archives

of thermodynamics

Vol. 38(2017), No. 2, 21-60

DOI: $10.1515 /$ aoter-2017-0009

\title{
On wave propagation in a random micropolar generalized thermoelastic medium
}

\author{
MANINDRA MITRA ${ }^{a}$ \\ RABINDRA KUMAR BHATTACHARYYA ${ }^{b *}$ \\ a Department of Mathematics, Presidency College, Kolkata 700073, India \\ $b$ Department of Applied Mathematics, Calcutta University Kolkata \\ 700009, India
}

\begin{abstract}
This paper endeavours to study aspects of wave propagation in a random generalized-thermal micropolar elastic medium. The smooth perturbation technique conformable to stochastic differential equations has been employed. Six different types of waves propagate in the random medium. The dispersion equations have been derived. The effects due to random variations of micropolar elastic and generalized thermal parameters have been computed. Randomness causes change of phase speed and attenuation of waves. Attenuation coefficients for high frequency waves have been computed. Second moment properties have been briefly discussed with application to wave propagation in the random micropolar elastic medium. Integrals involving correlation functions have been transformed to radial forms. A special type of generalized thermo-mechanical auto-correlation functions has been used to approximately compute effects of random variations of parameters. Uncoupled problem has been briefly outlined.
\end{abstract}

Keywords: Waves; Random; Micropolar; Thermoelastic; Propagation

\section{Introduction}

A large number of research papers covering various branches of theoretical and computational micropolar elasticity are being reported in the literature

${ }^{*}$ Corresponding Author. Email rabindrakb@yahoo.com 
every year in recent time. Eringen has published many pioneering papers and treatises on various aspects of micropolar elasticity. The coupled stress theory developed by Eringen comprises granular materials as also composite fibrous materials [1]. For this reason authors from different research fields have been taking interest in micropolar elasticity. The associated microrotational motions, spin, couple stress inertia, couple stress and distributed body couples were defined. Micropolar thermoelasticity also has become an important field of research these days. Eringen derived equations of motion, constitutive equations and boundary conditions for a class of micromorphic elastic solids whose microelements can undergo expansions or contractions defined as stretch [2]. He also defined and proposed a theory of thermomicrostretch fluids and bubbly liquids [3]. Microstretch and micropolar continua and all other aspects of micropolar studies can be found in Eringen's foundational treatise entitled Microcontinuum Field Theories [4]. Micropolar thermoelastcity and stretch have attracted the attention of many authors. Marin formulated some theorems on elastostatics of micropolar material with voids [5]. He investigated the behaviour of porous solids in which the matrix material is elastic and the interstices are voids of materials. In a more recent paper Marin [6] considered the concept of domain of influence in the context of displacement and microrotation fields along with the microstretch function. Marin and Lupu [7] on the other hand discussed the problem of harmonic vibrations of micropolar elastic materials under thermoelasticity. Marin and Marinescu [8] studied thermoelasticity of initially stressed bodies. Kumar [9] examined the problem of wave propagation in a micropolar viscoelastic medium under generalized thermoelasticity. Singh [10] studied plane wave propagation in a homogeneous transversely isotropic thermally conducting elastic solid with two relaxation times. Singh and Kumar [11] investigated the problem of reflection and refraction of plane waves at an interface between micropolar elastic solid and viscoelastic solid. Kumar and Deswal [12] discussed the problem of surface wave propagation in a micropolar thermoelastic medium. Kumar and Singh [13] studied effects of stretch in wave propagation in a micropolar material again under generalized thermoelasticity. Kumar and Tomar [14] studied aspects of reflected and refracted micropolar waves. Aouadi [15] considered a medium with a microstructure and derived general equations of motion and constitutive equations. On the other hand, Suiker, Borst and Chang [16] derived a second-gradient micro-polar constitutive theory on micro-mechanical modelling of granular medium. Majewski [17] dealt 
with seismic rotation waves in a micropolar elastic earth. In fact varieties of papers on wave propagation in micropolar elastic, micropolar thermoelastic and coupled micropolar elastic media are being frequently reported in the literature. It is not found necessary to cite more of these recent publications simply because they fall exclusively to the non-random domain.

The present paper instead proposes to focus on the procedure to evaluate effects of random variation of parameters of the inhomogeneous micropolar medium on propagation of waves and associated phenomena. To date none other than a single paper by Mitra and Bhattacharyya [18] has appeared in the literature dwelling on randomness in relation to waves in micropolar medium.

The present paper therefore aims at investigating wave propagation phenomenon in a random generalized thermal micropolar elastic medium, and associated statistical properties such as second moment and its application. The parameters representing inhomogeneities of the coupled medium are assumed to vary slightly from their mean values. The originality of the paper lies in attempting to measure effects of random variations of parameters on wave propagation in the micropolar generalized thermoelastic medium following procedures of the authors' earlier paper [18].

The heat conduction equation and the coupling model have been chosen under the generalized thermoelasticity proposed by Lord and Shulman [19] and Green-Lindsay [20]. The details of L-S and G-L theories of generalized thermoelasticity can be found in Ignaczak and Starzewski [21] and need not to be repeated here. Generalized thermoelasticity attempts at nullifying the physical anomaly of classical thermoelasticity which insists that thermal speed assumes infinite speed at infinity ([21], p. xii). Consequently two relaxation parameters $t_{0}, t_{1}$, appear in the generalized model heat conduction equation.

The methodology adopted is the smooth perturbation technique enunciated by Keller [22]. Karal and Keller [23], Keller and Karal [24], Chow [25], Chen and Tien [26] and many others used it in studying elastic, electromagnetic, thermal and other waves. Bhattacharyya $[27,28]$ and Bera [29] pursued the method in the study of wave propagation phenomena in the coupled media.

In fact the study of problems of wave propagation in random media flourished since the time of Chernov [30]. He first suggested adoption of an exponentially decaying form for a two-point dielectric correlation function. Beran and McCoy [31] proposed the technique of iterative perturbation in 
studying mean field variations in the dielectric and other media. Beran, Frankenthal, Deshmukh and Whitman studied propagation of radiation in time-dependent three-dimensional random media [32]. Sobczyk studied elastic wave propagation in a discrete random medium [33]. He developed a general formalism of the analysis of coherent elastic waves in terms of scatters. In fact, Wenzel [34], Sobczyk, Wedrychowicz and Spencer [35], Frankenthal and Beran [36], Uscinski [37] and Frisch [38] have made extensive studies on various aspects of randomness in physical sciences, wave propagation phenomena and random characteristics of media. In particular, Chen and Soong [39] worked on covariance properties of waves propagating in a medium. A detailed discussion on properties and applications of random differential equations can be found, among others, in the treatise of Soong [40]. Akira Ishimaru discussed the development of the theory of wave propagation and scattering in random media in his landmark treatise on the subject [41]. Choudhury, Basu and Bhattacharyya discussed the phenomenon of wave propagation in a rotating random granular medium under generalized thermoelasticity [42]. On the basis of these citations one cannot but admit the importance of the study of randomness in applied mathematics and physical sciences. The present paper however proposes to discuss randomness in respect of waves in a micropolar medium impressed by a generalized thermoelastic field.

\section{The problem}

Using the smooth perturbation technique [22] the field equations have been put in the form

$$
\begin{gathered}
L(\vec{x}, t) V(\vec{x}, t)=F(\vec{x}, t), \\
L=L_{0}+\varepsilon L_{1}+\varepsilon^{2} L_{2},
\end{gathered}
$$

where: $L$ - random linear operator, $V$ - field vector, $F$ - non-random source, $L_{0}$ - unperturbed part of $L, L_{1}, L_{2}$ - first and second order perturbation of $L$ respectively, and $\varepsilon$ - small parameter measuring the scale of random fluctuation of generalized thermo-micropolar elastic inhomogeneities of the medium.

Then employing iterative operations [23] it can be shown that the mean field $\langle V\rangle$ satisfies the equation

$$
\left[L_{0}+\varepsilon\left\langle L_{1}\right\rangle+\varepsilon^{2}\left\{\left\langle L_{2}\right\rangle+\left\langle L_{1}\right\rangle L_{0}^{-1}\left\langle L_{1}^{\prime}\right\rangle-\left\langle L_{1} L_{0}^{-1} L_{1}^{\prime}\right\rangle\right\}\right]\langle V\rangle=F
$$


with

$$
L_{0} G_{i j}\left(\vec{x}, \vec{x}^{\prime}, t, t^{\prime}\right)=\delta\left(\vec{x}, \vec{x}^{\prime}\right) \delta\left(t, t^{\prime}\right) \delta_{i j} .
$$

Here $\langle V(\vec{x}, t)\rangle$ is defined as the mean field quantity being the statistical average of the field vectors, an ensemble average. Also $G_{i j}$ is the appropriate Green's matrix (computed in the infinite domain) for $L_{0}, \delta\left(\vec{x}, \overrightarrow{x^{\prime}}\right)$ is the Dirac delta function and $\delta_{i j}$ is the Kronecker delta. Equation (3) is evidently an integro-differential equation, as in terms of $G_{0}, L_{0}^{-1}$ becomes

$$
L_{0}^{-1} f=\int G\left(\vec{x}, \overrightarrow{x^{\prime}}\right) f\left(\overrightarrow{x^{\prime}}\right) d \overrightarrow{x^{\prime}} .
$$

The present analysis aims at investigating the effects of random inhomogeneities on the propagation of waves in an interacting generalized thermomicropolar elastic medium. The equations of motion, constitutive equations and boundary conditions for the micropolar elastic field have been derived by Eringen [2]. The generalized thermal coupling is assumed to vary randomly only in the perturbed field. It may be recorded here that this assumption was necessitated to facilitate computation of the associated Green's matrix $[18,25,42]$. Therefore $L_{0}$ effectively represents the linear partial differential operator involving parameters of the unperturbed micropolar elastic medium only. Operator $L$ acts on the displacement vector $\vec{u}$, the microrotation vector $\vec{\phi}$ and $\theta$, the temperature. Six different types of body waves, which propagate in the non-random medium, depend upon the random inhomogeneities of the medium. The dispersion equations for these six longitudinal and transverse types of waves have been obtained. They involve terms up to the order of $\varepsilon^{2}$, since $\left\langle L_{1}\right\rangle \neq 0$ in this case. However, finally the effect of randomness comes to alter the wave number to the order of $\varepsilon^{2}$ only. Theoretically therefore the effect of randomness comes to be small to the order of $\varepsilon^{2}$. Deviations in the propagation constants from their unperturbed values have been calculated in terms of $\delta_{l}, \delta_{n}, \delta_{c}, \delta_{s}$. for different types of waves defined in the text in Sec. 4. These quantities involve auto- and cross-correlation functions between micropolar elastic and generalized thermal field parameters. Assuming simple auto-correlation functions only for density and two thermo-mechanical coupling parameters in the forms $[25,29]$

$$
R_{\rho \rho}(r)=\left\langle\rho_{1}^{2}\right\rangle e^{-\frac{r}{a}} \text { and } R_{m m}(r)=\left\langle\rho_{1}^{2}\right\rangle e^{-\frac{r}{b}}, R_{m m^{*}}(r) e^{-\frac{r}{b_{1}}},
$$

where $\left\langle\rho_{1}^{2}\right\rangle$ is the mean square value of the perturbation density $\rho_{1}$, and $a, b, b_{1}$ are correlation lengths of inhomogeneities, and all other parameters 
viz., $\lambda, \mu, \alpha$, etc., to be nonrandom, it has been shown that body waves for which the deviations are measured in terms of $\delta_{c}, \delta_{s}$, attenuate for high frequencies. It has been proved earlier [18] that second moments of the form $\left\langle\overline{\bar{v}}^{T} \bar{v}\right\rangle-\left\langle\overline{\bar{v}}^{T}\right\rangle\langle\bar{v}\rangle$ is a small quantity of the order of $\varepsilon$ only but certainly larger than the order of $\varepsilon^{2},(v$ being the solution of the equation $L v(\vec{x})=0$ referred to in Sec. 9). The same result applies in this case too. Components of associated Green's matrix which were computed earlier have been quoted in Appendix I as a ready reference. The various integrals viz., $a_{11}, b_{63}$, etc. involving correlation functions between different micropolar, elastic and generalized thermal parameters have been reduced to radial forms; these are presented in Sec. 6. In all these cases, lengthy and cumbersome computations become unavoidable. Radial transformations ensure symmetrical propagation of radiations in all directions in the infinite medium.

Field equations for a micropolar elastic medium under the influence of a generalized thermal field are written explicitly. The displacement equation of motion in the randomly varying inhomogeneous micropolar elastic medium is written following Eringen [1] and Chow [25] as

$$
\begin{aligned}
& (\lambda+\mu) \vec{\nabla}(\vec{\nabla} \cdot \vec{u})+(\mu+\kappa) \nabla^{2} \vec{u}+\vec{\nabla} \lambda(\vec{\nabla} \cdot \vec{u})+\vec{\nabla} \mu \times(\vec{\nabla} \times \vec{u}) \\
& +(\vec{\nabla}(2 \mu+\kappa) \cdot \vec{\nabla}) \vec{u}+\vec{\nabla} \times(\kappa \vec{\phi})-\vec{\nabla}\left[m\left\{\theta+t_{1} \dot{\theta}\right\}\right]+\vec{f}=\rho \ddot{\vec{u}} .
\end{aligned}
$$

The microrotation equation of motion is represented by [1]

$$
\begin{aligned}
& \kappa(\vec{\nabla} \times \vec{u})+(\alpha+\beta) \vec{\nabla}(\vec{\nabla} \cdot \vec{\phi})+(\vec{\nabla} \alpha(\vec{\nabla} \cdot \vec{\phi}))+\gamma \nabla^{2} \vec{\phi} \\
& +\vec{\nabla} \beta \times(\vec{\nabla} \times \vec{\phi})+\{\vec{\nabla}(\beta+\gamma) \cdot \vec{\nabla}\} \vec{\phi}-2 \kappa \vec{\phi}+\vec{l}=\rho j \overrightarrow{\vec{\phi}} .
\end{aligned}
$$

Finally the generalized thermal equation is written following Ignaczak and Ostoja-Starzewski [21], Bera [29], and Singh [10] as

$$
\eta\left[\dot{\theta}+t_{0} \ddot{\theta}\right]=\vec{\nabla} \cdot[\nu \vec{\nabla} \theta]-\theta_{0} m \vec{\nabla} \cdot\left[\dot{\vec{u}}+\delta_{l k} t_{0} \ddot{\vec{u}}\right]+q,
$$

where $\dot{\theta}=\frac{\theta(\vec{x}, t)}{\partial t}, \ddot{\theta}=\frac{\partial^{2} \theta(\vec{x}, t)}{\partial t^{2}}$, etc. Here 


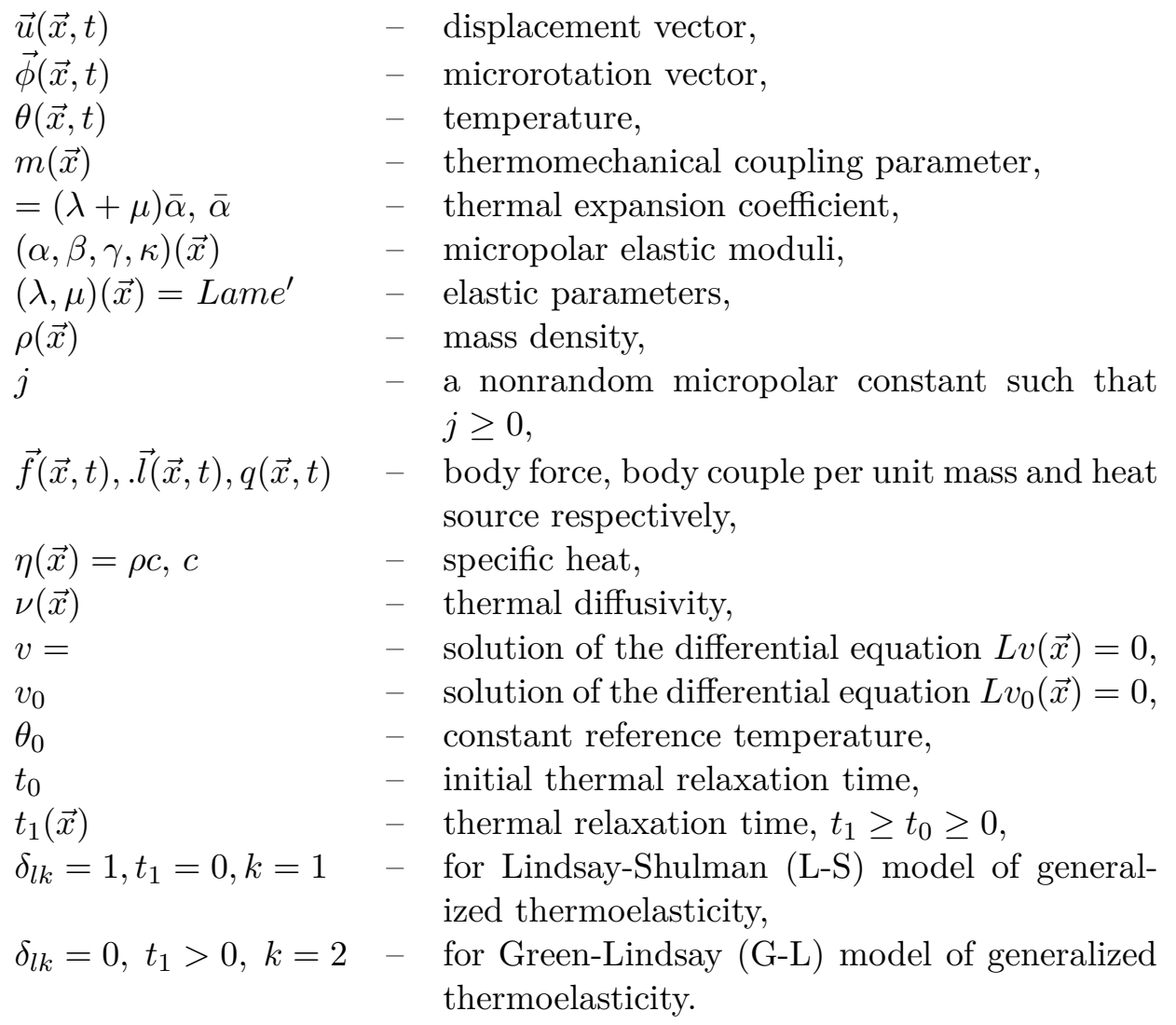

Let the differential operators $(M, N, R),(P, Q),(K, S)$ act respectively on $\vec{u}, \vec{\phi}$, and $\theta$. Then field Eqs. (5)-(7) can be put in matrix form:

$$
L V(\vec{x}, t)=\left[\begin{array}{ccc}
M & P & K \\
N & Q & 0 \\
R & 0 & S
\end{array}\right](\vec{x}, t)\left[\begin{array}{c}
\vec{u}(\vec{x}, t) \\
\vec{\phi}(\vec{x}, t) \\
T(\vec{x}, t)
\end{array}\right]=F(\vec{x}, t)=\left[\begin{array}{c}
\vec{f}(\vec{x}, t) \\
\vec{l}(\vec{x}, t) \\
q(\vec{x}, t)
\end{array}\right] .
$$

Differential operators $(M, P, K, N, Q, R, S)(\vec{x}, t)$ are explicitly defined as

$$
\begin{aligned}
M \vec{u}= & \rho \frac{\partial^{2} \vec{u}}{\partial t^{2}}-(\lambda+\mu) \vec{\nabla}(\vec{\nabla} \cdot \vec{u})-(\mu+\kappa) \nabla^{2} \vec{u}-\vec{\nabla} \lambda(\vec{\nabla} \cdot \vec{u}) \\
& -\vec{\nabla} \mu \times(\vec{\nabla} \times \vec{u})-(\vec{\nabla}(2 \mu+\kappa) \cdot \vec{\nabla}) \vec{u}, \\
P \vec{\phi}= & -\vec{\nabla} \times(\kappa \vec{\phi}), \\
K \theta= & \vec{\nabla}\left[m\left(\theta+t_{1} \frac{\partial \theta}{\partial t}\right)\right]=\vec{\nabla}(m \theta)+\vec{\nabla}\left(m t_{1} \frac{\partial \theta}{\partial t}\right)=\left[\vec{\nabla}(m \times)+\vec{\nabla}\left(m t_{1} \frac{\partial}{\partial t}\right)\right] \theta, \\
N \vec{u}= & -\kappa(\vec{\nabla} \times \vec{u}),
\end{aligned}
$$




$$
\begin{aligned}
Q \vec{\phi}= & \rho j \frac{\partial^{2} \vec{\phi}}{\partial t^{2}}-(\alpha+\beta) \vec{\nabla}(\vec{\nabla} \cdot \vec{\phi})-\vec{\nabla} \alpha(\vec{\nabla} \cdot \vec{\phi})-\gamma \nabla^{2} \vec{\phi}-\vec{\nabla} \beta \times(\vec{\nabla} \times \vec{\phi}) \\
& -(\vec{\nabla}(\beta+\gamma) \cdot \vec{\nabla}) \vec{\phi}+2 \kappa \vec{\phi}, \\
R \vec{u}= & \theta_{0} m \vec{\nabla} \cdot\left[\frac{\partial \vec{u}}{\partial t}+\delta_{l k} t_{0} \frac{\partial^{2} \vec{u}}{\partial t^{2}}\right]=\left[\theta_{0} m \vec{\nabla} \cdot\left(\frac{\partial}{\partial t}+\delta_{l k} t_{0} \frac{\partial^{2}}{\partial t^{2}}\right)\right] \vec{u}, \\
S \theta= & \eta\left(\frac{\partial \theta}{\partial t}+t_{0} \frac{\partial^{2} \theta}{\partial t^{2}}\right)-\vec{\nabla} \cdot(\nu \nabla \theta)=\left[\eta\left(\frac{\partial}{\partial t}+t_{0} \frac{\partial^{2}}{\partial t^{2}}\right)-\vec{\nabla} \cdot(\nu \vec{\nabla})\right] \theta .
\end{aligned}
$$

Next let us assume

$$
V(\vec{x}, t)=\left[\begin{array}{c}
\vec{u}(\vec{x}) \\
\vec{\phi}(\vec{x}) \\
\theta(\vec{x})
\end{array}\right] e^{-i \omega t}=V_{0}(\vec{x}) e^{-i \omega t},(\text { say })
$$

and

$$
F(\vec{x}, t)=\left[\begin{array}{c}
\vec{f}_{0}(\vec{x}) \\
\vec{l}_{0}(\vec{x}) \\
q_{0}(\vec{x})
\end{array}\right] e^{-i \omega t}=F_{0}(\vec{x}) e^{-i \omega t} .
$$

Here $\omega$ is the frequency of propagating wave and $i=\sqrt{-1}$. The mean field Eq. (3), involving the Green's matrix, can be put in the form

$$
\begin{aligned}
& {\left[L_{0}(\vec{x})+\varepsilon\left\langle L_{1}(\vec{x})\right\rangle+\varepsilon^{2}\left\{\left\langle L_{2}(\vec{x})\right\rangle+\left\langle L_{1}(\vec{x})\right\rangle L_{0}^{-1}\left(\left|\vec{x}-\vec{x}^{\prime}\right|\right)\left\langle L_{1}\left(\vec{x}^{\prime}\right)\right\rangle\right.\right.} \\
& \left.\left.-\left\langle L_{1}(\vec{x}) L_{0}^{-1}\left(\left|\vec{x}-\vec{x}^{\prime}\right|\right) L_{1}\left(\vec{x}^{\prime}\right)\right\rangle\right\}\right]\left\langle V_{0}\left(\vec{x}^{\prime}\right)\right\rangle=F_{0}
\end{aligned}
$$

where the associated Green's tensor is the kernel of the nonrandom operator equation represented by

$$
L_{0} G_{i j}\left(\vec{x}, \vec{x}^{\prime}\right)=\delta\left(\vec{x}, \vec{x}^{\prime}\right) \delta_{i j} .
$$

Then Eqs. (10) and (11) redefine operators $(M, P, K, N, Q, R, S)(\vec{x}, t)$ as $M=-\rho \omega^{2}-(\lambda+\mu) \vec{\nabla}(\vec{\nabla} \cdot)-(\mu+\kappa) \nabla^{2}-\vec{\nabla} \lambda(\vec{\nabla} \cdot)-\vec{\nabla} \mu \times(\vec{\nabla} \times)-(\vec{\nabla}(2 \mu+$

$\kappa) \cdot \vec{\nabla})$,

$P=-\vec{\nabla} \times(\kappa)$,

$K=\vec{\nabla}(m \times)-i \omega \vec{\nabla}\left(m t_{1}\right)=\vec{\nabla}(m \times)-i \omega \vec{\nabla}\left(m^{\bullet}\right), m^{\bullet}=m t_{1}($ say $)$,

$N=-\kappa(\vec{\nabla} \times)$ 


$$
\begin{aligned}
& Q=-\rho j \omega^{2}-(\alpha+\beta) \vec{\nabla}(\vec{\nabla} \cdot)-\vec{\nabla} \alpha(\vec{\nabla} \cdot)-\gamma \nabla^{2}-\vec{\nabla} \beta \times(\vec{\nabla} \times)-\{\vec{\nabla}(\beta+\gamma) \cdot \vec{\nabla}\}+2 \kappa \\
& \left.\left.R=-\omega \theta_{0} m \vec{\nabla} \cdot\left[i+\delta_{l k} t_{0} \omega\right)\right]=-i \omega \theta_{0}\left[1-i \delta_{l k} t_{0} \omega\right)\right] m(\vec{\nabla} \cdot), \\
& S=-i \omega\left(1-i t_{0} \omega\right) \eta-(\vec{\nabla} \cdot(\nu \nabla)) .
\end{aligned}
$$

\section{Solution}

Let us assume plane wave propagation in the medium and set

$$
\left[\begin{array}{c}
\vec{u}(\vec{x}) \\
\vec{\phi}(\vec{x}) \\
\theta(\vec{x})
\end{array}\right]=\left[\begin{array}{c}
\vec{A} \\
\vec{B} \\
C
\end{array}\right] e^{i \vec{k} \cdot \vec{x}}
$$

Here $\vec{k}$ indicates direction of the propagating wave and $|\vec{k}|=k$ is the wave number. Then the physical parameters $\lambda, \mu, \rho, \alpha, \beta, \gamma, \kappa, \eta, \nu, m$, and $m^{\bullet}=t_{1} m$, are random functions of $\vec{x}(x, y, z)$, and since random deviations are small, we write, following, Karal and Keller [22] and Chow [25]:

$$
\begin{aligned}
& \left(\lambda, \mu, \rho, \alpha, \beta, \gamma, \kappa, \eta, \nu, m, m^{\bullet}\right)=\left(\lambda_{0}, \mu_{0}, \rho_{0}, \alpha_{0}, \beta_{0}, \gamma_{0}, \kappa_{0}, \eta_{0}, \nu_{0},\right. \\
& \left.m_{0}=0, m_{0}^{\bullet}=0\right)+\varepsilon\left(\lambda_{1}, \mu_{1}, \rho_{1}, \alpha_{1}, \beta_{1}, \gamma_{1}, \kappa_{1}, \eta_{1}, \nu_{1}, m_{1}, m_{1}^{\bullet}\right)(\vec{x}),
\end{aligned}
$$

where $\left(\lambda_{0}, \ldots ., \nu_{0}\right)$ are constants and $\left(\lambda_{1}, \ldots . ., \nu_{1}, m_{1}, m_{1}^{\bullet}\right)$ are functions of $\vec{x}$ representing random fluctuations of the corresponding quantities such that

$$
\left\langle\lambda_{1}, \ldots \ldots, \nu_{1}\right\rangle=0 \text {. }
$$

Conditions (16) and (17) ensure that fluctuations from nonrandom values of parameters remain small. It is further assumed that

$$
\begin{gathered}
m=\varepsilon m_{1}(\vec{x}), \text { such that }\left\langle m_{1}(\vec{x})\right\rangle=m_{2},(\text { say }), m_{2} \neq 0, \\
\text { and } m^{\bullet}=\varepsilon m_{1}^{\bullet}(\vec{x}), \text { such that }\left\langle m_{1}^{\bullet}(\vec{x})\right\rangle=m_{3},(\text { say }), m_{3} \neq 0 .
\end{gathered}
$$

The last two assumptions confirm that the generalized thermal field is taken to be weakly random. A new parameter $m^{\bullet}(\vec{x})=\left(t_{1} m\right)(\vec{x})$, is introduced characterizing the random thermal coupling with the micropolar elastic medium. By the help of Eqs. (16)-(18) one gets

$$
L_{0}=\left[\begin{array}{lll}
M_{0} & P_{0} & 0 \\
N_{0} & Q_{0} & 0 \\
0 & 0 & S_{0}
\end{array}\right],
$$




$$
L_{1}(\vec{x})=\left[\begin{array}{ccc}
M_{1} & P_{1} & K_{1} \\
N_{1} & Q_{1} & 0 \\
R_{1} & 0 & S_{1}
\end{array}\right](\vec{x})
$$

and

$$
\begin{aligned}
& M_{0}=-\rho_{0} \omega^{2}-\left(\lambda_{0}+\mu_{0}\right) \vec{\nabla}(\vec{\nabla} \cdot)-\left(\mu_{0}+\kappa_{0}\right) \nabla^{2}, \\
& P_{0}=-\kappa_{0}(\vec{\nabla} \times)=N_{0}, \\
& Q_{0}=\left(2 \kappa_{0}-\rho_{0} j \omega^{2}\right)-\left(\alpha_{0}+\beta_{0}\right) \vec{\nabla}(\vec{\nabla} \cdot)-\gamma_{0} \nabla^{2}, \\
& S_{0}=-i \omega\left(1-i t_{0} \omega\right) \eta_{0}-\nu_{0} \nabla^{2} .
\end{aligned}
$$

The deterministic operators save $S_{0}$ are independent of generalized thermal relaxation parameters and the thermo-mechanical parameters. However the perturbed operators $K_{1}, R_{1}, S_{1}$, are characterized by $m_{1}, m_{1}^{\bullet}, t_{0}, t_{1}$, such that

$$
\begin{aligned}
& M_{1}=-\rho_{1} \omega^{2}-\left(\lambda_{1}+\mu_{1}\right) \vec{\nabla}(\vec{\nabla} \cdot)-\left(\mu_{1}+\kappa_{1}\right) \nabla^{2}-\vec{\nabla} \lambda_{1}(\vec{\nabla} \cdot)-\vec{\nabla} \mu_{1} \times(\vec{\nabla} \times)- \\
& \left\{\vec{\nabla}\left(2 \mu_{1}+\kappa_{1}\right) \cdot \vec{\nabla}\right\} \\
& P_{1}=-\vec{\nabla} \times\left(\kappa_{1}\right), \\
& K_{1}=\vec{\nabla}\left(m_{1}\right)-i \omega \vec{\nabla} m_{1}^{\bullet}, \\
& N_{1}=-\kappa_{1}(\vec{\nabla} \times), \\
& Q_{1}=-\rho_{1} j \omega^{2}-\left(\alpha_{1}+\beta_{1}\right) \vec{\nabla}(\vec{\nabla} \cdot)-\vec{\nabla} \alpha_{1}(\vec{\nabla} \cdot)-\gamma_{1} \nabla^{2}-\vec{\nabla} \beta_{1} \times(\vec{\nabla} \times)-\left(\vec { \nabla } \left(\beta_{1}+\right.\right. \\
& \left.\left.\gamma_{1}\right) \cdot \vec{\nabla}\right)+2 \kappa_{1}, \\
& R_{1}=-i \omega \theta_{0}\left(1-i \delta_{l k} t_{0} \omega\right) m_{1}(\vec{\nabla} \cdot), \\
& S_{1}=-i \omega\left(1-i t_{0} \omega\right) \eta_{1}-\left\{\vec{\nabla} \cdot\left(\nu_{1} \nabla\right)\right\} .
\end{aligned}
$$

A computational note is given below:

$$
\begin{aligned}
S & =-i \omega\left(1-i t_{0} \omega\right) \eta-(\vec{\nabla} \cdot(\nu \nabla) \\
& =-i \omega\left(1-i t_{0} \omega\right)\left(\eta_{0}+\varepsilon \eta_{1}\right)-\vec{\nabla} \cdot\left[\left(\nu_{0}+\varepsilon \nu_{1}\right) \vec{\nabla}\right] \\
& =-i \omega\left(1-i t_{0} \omega\right) \eta_{0}-\vec{\nabla} \cdot\left(\nu_{0} \vec{\nabla}\right)-\varepsilon i \omega\left(1-i t_{0} \omega\right) \eta_{1}-\varepsilon \vec{\nabla} \cdot\left(\nu_{1} \vec{\nabla}\right) \\
& =\left\{-i \omega\left(1-i t_{0} \omega\right) \eta_{0}-\nu_{0} \nabla^{2}\right]+\left\{-\varepsilon i \omega\left(1-i t_{0} \omega\right) \eta_{1} \varepsilon \vec{\nabla} \cdot\left(\nu_{1} \vec{\nabla}\right)\right\} \\
& \left.=S_{0}+\varepsilon S_{1}\right] .
\end{aligned}
$$

By virtue of Eqs. (17)-(18) one obtains

$$
\left\langle L_{1}\right\rangle=\left[\begin{array}{lrcc}
0 & 0 & \left(m_{2}-i \omega m_{3}\right) \vec{\nabla} \\
0 & 0 & 0 \\
-i \omega \theta_{0}\left(1-i \delta_{l k} t_{0} \omega\right) m_{2}(\vec{\nabla} \cdot) & 0 & 0
\end{array}\right] \neq 0,
$$


where

$$
m_{2}=\left\langle m_{1}(\vec{x})\right\rangle=\text { const. } \neq 0, m_{3}=\left\langle m_{1}^{\bullet}(\vec{x})\right\rangle=\text { const. } \neq 0 \text { and }\left\langle L_{2}\right\rangle=0 .
$$

Thus Eq. (3) involves first and second perturbation terms; with vanishing body force, body couple and the heat source, Eq. (12) reduces to

$$
\begin{aligned}
& {\left[L_{0}(\vec{x})+\varepsilon\left\langle L_{1}(\vec{x})\right\rangle+\varepsilon^{2}\left\{\left\langle L_{1}(\vec{x})\right\rangle L_{0}^{-1}\left(\left|\vec{x}-\vec{x}^{\prime}\right|\right)\left\langle L_{1}\left(\vec{x}^{\prime}\right)\right\rangle\right.\right.} \\
& \left.\left.-\left\langle L_{1}(\vec{x}) L_{0}^{-1}\left(\left|\vec{x}-\vec{x}^{\prime}\right|\right) L_{1}\left(\vec{x}^{\prime}\right)\right\rangle\right\}\right]\left\langle V_{0}\left(\vec{x}^{\prime}\right)\right\rangle=0 .
\end{aligned}
$$

The components of Green's tensor corresponding to $L_{0}^{-1}$ are given by (say),

$$
G_{i j}=\left[\begin{array}{ccc}
G_{0} & G_{1} & 0 \\
G_{2} & G_{3} & 0 \\
0 & 0 & G_{4}
\end{array}\right] .
$$

The components $G_{0}(r), G_{1}(r), G_{2}(r)$, and $G_{3}(r)$ have already been evaluated [18] and have been given in the Appendix I. The component $G_{4}(r)$ is defined by

$$
\nabla^{2} G_{4}(r)+\frac{i \omega\left(1-i \omega t_{0}\right) \eta_{0}}{\nu_{0}} G_{4}(r)=\delta\left(\vec{x}-\vec{x}^{\prime}\right) \delta_{i j}
$$

Hence

where

$$
G_{4}(r)=-\frac{e^{i \beta r}}{4 \pi r} I_{3}
$$

$$
\beta=\left[\frac{i \omega\left(1-i \omega t_{0}\right) \eta_{0}}{\nu_{0}}\right]^{\frac{1}{2}},
$$

and $I_{3}$ is the identity matrix.

Hence

$$
\begin{aligned}
\beta & =\sqrt{\frac{\omega \eta_{0}}{2 \nu_{0}}}\left\{\left[\sqrt{\omega^{2} t_{0}^{2}-1}+\omega t_{0}\right]^{\frac{1}{2}}+i\left[\sqrt{\omega^{2} t_{0}^{2}-1}-\omega t_{0}\right]^{\frac{1}{2}}\right\} \\
& =\beta_{1}+i \beta_{2}(\text { say }) .
\end{aligned}
$$

It is observed that $G_{i}(r), i=0,1,2,3$ are independent of thermo-mechanical coupling parameter $m(\vec{x})$ and generalized thermoelastic relaxation times $t_{0}, t_{1}$, and $m^{\bullet}(\vec{x})$. On the other hand $G_{4}(r)$ is independent of $m(\vec{x})$ but depends upon the generalized thermoelastic relaxation time $t_{0}$. Substituting (15) in the mean field Eq. (25) one gets the following three vector equations: 


$$
\begin{aligned}
& {\left[\rho_{0} \omega^{2} \vec{A}-\left(\lambda_{0}+\mu_{0}\right)(\vec{k} \cdot \vec{A}) \vec{k}-\left(\mu_{0}+\kappa_{0}\right) k^{2} \vec{A}\right]+i \kappa_{0}(\vec{k} \times \vec{B})-\varepsilon\left(i m_{2}+\omega m_{3}\right) C \vec{k}} \\
& +\varepsilon^{2}\left[\omega \theta_{0}\left(1-\omega t_{0} \delta_{l k}\right) m_{2}\left(m_{2}-i \omega m_{3}\right)(\vec{k} \cdot \vec{A})\right] \int\left[\left(\vec{\nabla} G_{4}\right)\right] e^{-i \vec{k} \cdot \vec{r}} d \vec{r} \\
& -\varepsilon^{2} \int\left[\begin{array}{l}
\left\langle\left[ M_{1}\left\{G_{0}\left(M_{1}^{\prime} \vec{A}+P_{1}^{\prime} \vec{B}+K_{1}^{\prime} C\right)+G_{1}\left(N_{1}^{\prime} \vec{A}+Q_{1}^{\prime} \vec{B}\right)\right\}\right.\right. \\
+P_{1}\left\{G_{2}\left(M_{1}^{\prime} \vec{A}+P_{1}^{\prime} \vec{B}+K_{1}^{\prime} C\right)+G_{3}\left(N_{1}^{\prime} \vec{A}+Q_{1}^{\prime} \vec{B}\right)\right\} \\
\left.\left.+K_{1} G_{4}\left(R_{1}^{\prime} \vec{A}+S_{1}^{\prime} C\right)\right]\right\rangle
\end{array}\right] e^{-i \vec{k} \cdot \vec{r}} d \vec{r}=0
\end{aligned}
$$

$$
\begin{aligned}
& i \kappa_{0}(\vec{k} \times \vec{A})-\left[\left(2 \kappa_{0}-\rho_{0} j \omega^{2}+\gamma_{0} k^{2}\right) \vec{B}+\left(\alpha_{0}+\beta_{0}\right)(\vec{k} \cdot \vec{B}) \vec{k}\right] \\
& -\varepsilon^{2} \int\left[\begin{array}{l}
\left\langle\left[ N_{1}\left\{G_{0}\left(M_{1}^{\prime} \vec{A}+P_{1}^{\prime} \vec{B}+K_{1}^{\prime} C\right)+G_{1}\left(N_{1}^{\prime} \vec{A}+Q_{1}^{\prime} \vec{B}\right)\right\}\right.\right. \\
\left.\left.+Q_{1}\left\{G_{2}\left(M_{1}^{\prime} \vec{A}+P_{1}^{\prime} \vec{B}+K_{1}^{\prime} C\right)+G_{3}\left(N_{1}^{\prime} \vec{A}+Q_{1}^{\prime} \vec{B}\right)\right\}\right]\right\rangle
\end{array}\right] e^{-i \vec{k} \cdot \vec{r}} d \vec{r}=0,
\end{aligned}
$$

and

$$
\begin{aligned}
& {\left[i \omega\left(1-i t_{0} \omega\right) \eta_{0}-\nu_{0} k^{2}\right] C-\varepsilon \omega \theta_{0}\left(1-i \omega t_{0} \delta_{l k}\right) m_{2}(\vec{k} \cdot \vec{A})} \\
& +\varepsilon^{2}\left[\omega \theta_{0}\left(1-i \omega t_{0} \delta_{l k}\right) m_{2}\left(m_{2}-i \omega m_{3}\right)\right] C \int\left[\left(\vec{\nabla} \cdot G_{0} \vec{k}\right)\right] e^{-i \vec{k} \cdot \vec{r}} d \vec{r} \\
& -\varepsilon^{2} \int\left[\begin{array}{l}
\left\langle\left[ R_{1}\left\{G_{0}\left(M_{1}^{\prime} \vec{A}+P_{1}^{\prime} \vec{B}+K_{1}^{\prime} C\right)+G_{1}\left(N_{1}^{\prime} \vec{A}+Q_{1}^{\prime} \vec{B}\right)\right\}\right.\right. \\
\left.\left.+S_{1} G_{4}\left(R_{1}^{\prime} \vec{A}+S_{1}^{\prime} C\right)\right]\right\rangle
\end{array}\right] e^{-i \vec{k} \cdot \vec{r}} d \vec{r}=0 .
\end{aligned}
$$

Eliminating $C$ from (29) and (30) with the help of (31) one gets the following two equations:

$$
\begin{aligned}
& {\left[\rho_{0} \omega^{2} \vec{A}-\left(\lambda_{0}+\mu_{0}\right)(\vec{k} \cdot \vec{A}) \vec{k}-\left(\mu_{0}+\kappa_{0}\right) k^{2} \vec{A}\right]+i \kappa_{0}(\vec{k} \times \vec{B})} \\
& -\varepsilon^{2} \frac{\omega \theta_{0}\left(1-i \omega t_{0} \delta_{l k}\right) m_{2}\left(i m_{2}+\omega m_{3}\right)(\vec{k} \cdot \vec{A})}{i \omega\left(1-i t_{0} \omega\right) \eta_{0}-\nu_{0} k^{2}} \vec{k} \\
& +\varepsilon^{2}\left[\omega \theta_{0}\left(1-\omega t_{0} \delta_{l k}\right) m_{2}\left(m_{2}-i \omega m_{3}\right)(\vec{k} \cdot \vec{A})\right] \int\left[\left(\vec{\nabla} G_{4}\right)\right] e^{-i \vec{k} \cdot \vec{r}} d \vec{r} \\
& -\varepsilon^{2} \int\left[\begin{array}{l}
{\left[\left\langle M_{1} G_{0} M_{1}^{\prime}\right\rangle \vec{A}+\left\langle M_{1} G_{0} P_{1}^{\prime}\right\rangle \vec{B}+\left\langle M_{1} G_{1} N_{1}^{\prime}\right\rangle \vec{A}\right.} \\
+\left\langle M_{1} G_{1} Q_{1}^{\prime}\right\rangle \vec{B}+\left\langle P_{1} G_{2} M_{1}^{\prime}\right\rangle \vec{A}+\left\langle P_{1} G_{2} P_{1}^{\prime}\right\rangle \vec{B} \\
\left.+\left\langle P_{1} G_{3} N_{1}^{\prime}\right\rangle \vec{A}+\left\langle P_{1} G_{3} Q_{1}^{\prime}\right\rangle \vec{B}+\left\langle K_{1} G_{4} R_{1}^{\prime}\right\rangle \vec{A}\right]
\end{array}\right] e^{-i \vec{k} \cdot \vec{r} d \vec{r}=0}
\end{aligned}
$$

and 


$$
\begin{aligned}
& i \kappa_{0}(\vec{k} \times \vec{A})-\left[\left(2 \kappa_{0}-\rho_{0} j \omega^{2}+\gamma_{0} k^{2}\right) \vec{B}+\left(\alpha_{0}+\beta_{0}\right)(\vec{k} \cdot \vec{B}) \vec{k}\right] \\
& -\varepsilon^{2} \int\left[\begin{array}{l}
\left\langle N_{1} G_{0} M_{1}^{\prime}\right\rangle \vec{A}+\left\langle N_{1} G_{0} P_{1}^{\prime}\right\rangle \vec{B}+\left\langle N_{1} G_{1} N_{1}^{\prime}\right\rangle \vec{A} \\
+\left\langle N_{1} G_{1} Q_{1}^{\prime}\right\rangle \vec{B}+\left\langle Q_{1} G_{2} M_{1}^{\prime}\right\rangle \vec{A}+\left\langle Q_{1} G_{2} P_{1}^{\prime}\right\rangle \vec{B} \\
+\left\langle Q_{1} G_{3} N_{1}^{\prime}\right\rangle \vec{A}+\left\langle Q_{1} G_{3} Q_{1}^{\prime}\right\rangle \vec{B}
\end{array}\right] e^{-i \vec{k} \cdot \vec{r}} d \vec{r}=0 .
\end{aligned}
$$

Some computations of the form

$$
\left\langle M_{1} G_{o} M_{1}^{\prime}\right\rangle \vec{A}=e^{-i \vec{k} \cdot \vec{x}^{\prime}}\left\langle M_{1} G_{o} M_{1}^{\prime}\right\rangle\left(\vec{A} e^{i \vec{k} \cdot \vec{x}^{\prime}}\right),
$$

are shown in the Appendix II. The integral in the third term of (32)

$$
\begin{gathered}
=\int\left[\vec{\nabla}\left(G_{4}(r)\right] e^{-i \vec{k} \cdot \vec{r}} d \vec{r}\right. \\
=-i \hat{k} \int_{0}^{\propto} e^{i \beta r} \sin k r d r, \beta=\beta_{1}+i \beta_{2}, \beta_{1}=\operatorname{Re} \beta, \beta_{2}=\operatorname{Im} \beta . \\
=\frac{-i \hat{k}}{2}\left[\frac{\beta_{1}+k}{\beta_{2}^{2}+\left(\beta_{1}+k\right)^{2}}-\frac{\beta_{1}-k}{\beta_{2}^{2}+\left(\beta_{1}-k\right)^{2}}\right. \\
\left.+i \beta_{2}\left(\frac{1}{\beta_{2}^{2}+\left(\beta_{1}-k\right)^{2}}-\frac{1}{\beta_{2}^{2}+\left(\beta_{1}+k\right)^{2}}\right)\right] .
\end{gathered}
$$

Hence (32) can be rewritten as

$$
\begin{aligned}
& {\left[\rho_{0} \omega^{2} \vec{A}-\left(\lambda_{0}+\mu_{0}\right)(\vec{k} \cdot \vec{A}) \vec{k}-\left(\mu_{0}+\kappa_{0}\right) k^{2} \vec{A}\right]+i \kappa_{0}(\vec{k} \times \vec{B})} \\
& -\varepsilon^{2} \frac{\omega \theta_{0}\left(1-i \omega t_{0} \delta_{l k}\right) m_{2}\left(i m_{2}+\omega m_{3}\right)(\vec{k} \cdot \vec{A})}{i \omega\left(1-i t_{0} \omega\right) \eta_{0}-\nu_{0} k^{2}} \vec{k} \\
& -\varepsilon^{2}\left[\omega \theta_{0}\left(1-\omega t_{0} \delta_{l k}\right) m_{2}\left(i m_{2}+\omega m_{3}\right)(\vec{k} \cdot \vec{A}) \widehat{k}\right] \int_{0}^{\infty} e^{i \beta r} \sin k r d r \\
& -\varepsilon^{2} \int\left[\begin{array}{c}
{\left[\left\langle M_{1} G_{0} M_{1}^{\prime}\right\rangle \vec{A}+\left\langle M_{1} G_{0} P_{1}^{\prime}\right\rangle \vec{B}+\left\langle M_{1} G_{1} N_{1}^{\prime}\right\rangle \vec{A}\right.} \\
+\left\langle M_{1} G_{1} Q_{1}^{\prime}\right\rangle \vec{B}+\left\langle P_{1} G_{2} M_{1}^{\prime}\right\rangle \vec{A}+\left\langle P_{1} G_{2} P_{1}^{\prime}\right\rangle \vec{B} \\
\left.+\left\langle P_{1} G_{3} N_{1}^{\prime}\right\rangle \vec{A}+\left\langle P_{1} G_{3} Q_{1}^{\prime}\right\rangle \vec{B}+\left\langle K_{1} G_{4} R_{1}^{\prime}\right\rangle \vec{A}\right]
\end{array}\right] e^{-i \vec{k} \cdot \vec{r}} d \vec{r}=0 .
\end{aligned}
$$

Thus we are left with two vector Eqs. (36) and (33), determining the propagation of waves in the interacting random medium. It is to be noted that none of these equations include any term of the order of $\varepsilon$ even though $\left\langle L_{1}\right\rangle\left\langle V_{0}(\vec{x})\right\rangle \neq 0$. Effects of randomness therefore is small to the order of $\varepsilon^{2}$. 


\section{Analysis of first perturbation dispersion equations}

We get back to considering Eqs. (29)-(31) to $\varepsilon$-order terms only, assuming without loss of generality:

$$
\vec{k}=(k, 0,0), \quad \vec{A}=\left(A_{1}, A_{2}, 0\right), \quad \vec{B}=\left(B_{1}, B_{2}, B_{3}\right) .
$$

Hence (29)-(31) reduce to

$$
\begin{aligned}
& {\left[\rho_{0} \omega^{2} \vec{A}-\left(\lambda_{0}+\mu_{0}\right)(\vec{k} \cdot \vec{A}) \vec{k}-\left(\mu_{0}+\kappa_{0}\right) k^{2} \vec{A}\right]+i \kappa_{0}(\vec{k} \times \vec{B})-\varepsilon\left(i m_{2}+\omega m_{3}\right) C \vec{k}=0,} \\
& i \kappa_{0}(\vec{k} \times \vec{A})-\left[\left(2 \kappa_{0}-\rho_{0} j \omega^{2}+\gamma_{0} k^{2}\right) \vec{B}+\left(\alpha_{0}+\beta_{0}\right)(\vec{k} \cdot \vec{B}) \vec{k}\right]=0, \\
& \text { and } \\
& {\left[i \omega\left(1-i t_{0} \omega\right) \eta_{0}-\nu_{0} k^{2}\right] C-\varepsilon \omega \theta_{0}\left(1-i \omega t_{0} \delta_{l k}\right) m_{2}(\vec{k} \vec{A})=0 .}
\end{aligned}
$$

Eliminating $C$ as before from Eq. (38) one gets

$$
\begin{aligned}
& {\left[\rho_{0} \omega^{2} \vec{A}-\left(\lambda_{0}+\mu_{0}\right)(\vec{k} \cdot \vec{A}) \vec{k}-\left(\mu_{0}+\kappa_{0}\right) k^{2} \vec{A}\right]+i \kappa_{0}(\vec{k} \times \vec{B})} \\
& -\varepsilon^{2}\left(i m_{2}+\omega m_{3}\right) \frac{\omega \theta_{0}\left(1-i \omega t_{0} \delta_{l k}\right) m_{2}(\vec{k} \cdot \vec{A})}{i \omega\left(1-i t_{0} \omega\right) \eta_{0}-\nu_{0} k^{2}} \vec{k}=0 .
\end{aligned}
$$

Thus (39) and (41) represent two dispersion equations. Terms to the order $\varepsilon$ however disappears from these equations. The equations almost reduce to two deterministic equations except for the presence of a second perturbation term in (41) inducing a weak thermoelastic influence on propagation of waves. These equations lead to the following six equations:

$$
\begin{gathered}
{\left[\rho_{0} \omega^{2}-\left(\lambda_{0}+2 \mu_{0}+\kappa_{0}\right) k^{2}\right] A_{1}-\varepsilon^{2}\left(i m_{2}+\omega m_{3}\right) \frac{\omega \theta_{0}\left(1-i \omega t_{0} \delta_{l k}\right) m_{2} k^{2}}{i \omega\left(1-i t_{0} \omega\right) \eta_{0}-\nu_{0} k^{2}} A_{1}=0} \\
{\left[\rho_{0} \omega^{2}-\left(\mu_{0}+\kappa_{0}\right) k^{2}\right] A_{2}+i \kappa_{0} k B_{3}=0} \\
i \kappa_{0} k B_{2}=0 .
\end{gathered}
$$

This last equation indicates

$$
B_{2}=0 \text {. }
$$

This indicates that microrotation waves do not propagate along the direction of $B_{2}$. Also Eq. (39) gives three Eqs. (45), (46), and (47):

$$
\left.\left(2 \kappa_{0}-\rho_{0} j \omega^{2}+\gamma_{0} k^{2}\right)+\left(\alpha_{0}+\beta_{0}\right) k^{2}\right] B_{1}=0
$$

and

$$
\left(2 \kappa_{0}-\rho_{0} j \omega^{2}+\gamma_{0} k^{2}\right) B_{2}=0 .
$$


This indicates that $B_{2}=0$, but

$$
2 \kappa_{0}-\rho_{0} j \omega^{2}+\gamma_{0} k^{2} \neq 0
$$

This confirms that there is no propagation along the direction of $B_{2}$. The third equation is

$$
i \kappa_{0} k A_{2}-\left(2 \kappa_{0}-\rho_{0} j \omega^{2}+\gamma_{0} k^{2}\right) B_{3}=0 .
$$

The dispersion equation for longitudinal waves propagating along the direction of $\vec{k}$ is represented by (42), that is, by

$$
\left[\rho_{0} \omega^{2}-\left(\lambda_{0}+2 \mu_{0}+\kappa_{0}\right) k^{2}\right]-\varepsilon^{2}\left(i m_{2}+\omega m_{3}\right) \frac{\omega \theta_{0}\left(1-i \omega t_{0} \delta_{l k}\right) m_{2} k^{2}}{i \omega\left(1-i t_{0} \omega\right) \eta_{0}-\nu_{0} k^{2}}=0 .
$$

This is a fourth degree equation in the wave number $k$. From Eq. (48) effects of randomness of the generalized thermal field determined by the presence of $m_{2}$, and $m_{3}$ is discernible to terms to the order of $\varepsilon^{2}$.

Equation (45) determines the propagation of microrotation waves along the direction of $B_{1}$; the wave number is determined by

$$
k^{2}=\frac{\rho_{0} j \omega^{2}-2 \kappa_{0}}{\alpha_{0}+\beta_{0}+\gamma_{0}} .
$$

Waves having amplitudes $A_{2}$ and $B_{3}$ are coupled by Eqs. (43) and (47). Eliminating $A_{2}$ and $B_{3}$ one gets the dispersion equation for the propagation of coupled waves in the form

$$
\frac{A_{2}}{B_{3}}=-\frac{i \kappa_{0} k}{\rho_{0} \omega^{2}-\left(\mu_{0}+\kappa_{0}\right) k^{2}}=\frac{2 \kappa_{0}-\rho_{0} j \omega^{2}+\gamma_{0} k^{2}}{i \kappa_{0} k},
$$

or

$$
\left[\rho_{0} \omega^{2}-\left(\mu_{0}+\kappa_{0}\right) k^{2}\right]\left[2 \kappa_{0}-\rho_{0} j \omega^{2}+\gamma_{0} k^{2}\right]=\kappa_{0}^{2} k^{2} .
$$

This equation which is again a fourth degree equation in the wave number $k$ however determines the dispersion equation of waves propagating in the non-thermal micropolar elastic medium. Equation (48) only includes one $\varepsilon^{2}$-order term which represents small generalized thermal effects. Equations (48), (49), and (50) can easily be solved numerically. 


\section{Analysis of dispersion equations}

We proceed to analyze dispersion Eqs. (33) and (36). Each term of the form $\int\left\langle M_{1} G_{0} M_{1}^{\prime}\right\rangle \vec{A} e^{i \vec{k} \cdot \vec{x}^{\prime}}$ contains linear functions of the displacement amplitudes, $A_{1}, A_{2}, A_{3}$, and the microrotation amplitudes $B_{1}, B_{2}, B_{3}$. Each of the integrals involves correlation functions between different micropolar elastic and generalized thermal parameters including the generalized thermomechanical coupling parameters $m$ and $m^{\bullet}$. It is observed that none of the integrands of the forms $\left\langle M_{1} G_{0} M_{1}^{\prime}\right\rangle \vec{A} e^{i \vec{k} \cdot \vec{x}^{\prime}}$ contains the perturbed operator $S_{1}$. Thus wave propagation in the coupled random medium is independent of the perturbation in the classical thermal parameters $\eta(\vec{x})$ and $\nu(\vec{x})$. Moreover the only integrand representing effects of random variation of generalized thermal field is $\left\langle K_{1} G_{4} R_{1}^{\prime}\right\rangle \vec{A} e^{i \vec{k} \cdot \vec{x}^{\prime}}$. The integrand involves the random coupling variable $m^{\bullet}$. In a latter section we will analyze (33) and (36) retaining the integral $\int\left\langle K_{1} G_{4} R_{1}^{\prime}\right\rangle \vec{A} e^{i \vec{k} \cdot \vec{x}^{\prime}} d \vec{x}^{\prime}$ and making all other correlation functions vanish.

Now equations (36) and (33) give rise to six equations. Then terms of the order $\varepsilon^{2}$ in each of these equations are arranged as linear combinations of five amplitudes $A_{1}, A_{2}, B_{1}, B_{2}, B_{3}$, the coefficients of each of which being integrals involving different correlation functions. Rewriting one gets:

$$
\begin{gathered}
{\left[\rho_{0} \omega^{2}-\left(\lambda_{0}+2 \mu_{0}+\kappa_{0}\right) k^{2}\right] A_{1}-\varepsilon^{2} \frac{\omega \theta_{0}\left(1-i \omega t_{0} \delta_{l k}\right) m_{2}\left(i m_{2}+\omega m_{3}\right) k^{2}}{i \omega\left(1-i t_{0} \omega\right) \eta_{0}-\nu_{0} k^{2}} A_{1}} \\
-\varepsilon^{2} \omega \theta_{0}\left(1-i \omega t_{0} \delta_{l k}\right) m_{2}\left(i m_{2}+\omega m_{3}\right) k A_{1} \int_{0}^{\infty} e^{i \beta r} \sin k r d r \\
-\varepsilon^{2}\left[a_{11} A_{1}+a_{12} A_{2}+b_{11} B_{1}+b_{12} B_{2}+b_{13} B_{3}\right]=0 \\
{\left[\rho_{0} \omega^{2}-\left(\mu_{0}+\kappa_{0}\right) k^{2}\right] A_{2}-i \kappa_{0} k B_{3}} \\
-\varepsilon^{2}\left[a_{21} A_{1}+a_{22} A_{2}+b_{21} B_{1}+b_{22} B_{2}+b_{23} B_{3}\right]=0 \\
i \kappa_{0} k B_{2}-\varepsilon^{2}\left[a_{31} A_{1}+a_{32} A_{2}+b_{31} B_{1}+b_{32} B_{2}+b_{33} B_{3}\right]=0 \\
{\left[\rho_{0} j \omega^{2}-2 \kappa_{0}-\left(\alpha_{0}+\beta_{0}+\gamma_{0}\right) k^{2}\right] B_{1}} \\
-\varepsilon^{2}\left[a_{41} A_{1}+a_{42} A_{2}+b_{41} B_{1}+b_{42} B_{2}+b_{43} B_{3}\right]=0 \\
\left(\rho_{0} j \omega^{2}-2 \kappa_{0}-\gamma_{0} k^{2}\right) B_{2}-\varepsilon^{2}\left[a_{51} A_{1}+a_{52} A_{2}+b_{51} B_{1}+b_{52} B_{2}+b_{53} B_{3}\right]=0 \\
i \kappa_{0} k A_{2}+\left(\rho_{0} j \omega^{2}-2 \kappa_{0}-\gamma_{0} k^{2}\right) B_{3} \\
-\varepsilon^{2}\left[a_{61} A_{1}+a_{62} A_{2}+b_{61} B_{1}+b_{62} B_{2}+b_{63} B_{3}\right]=0
\end{gathered}
$$


The integrands in the coefficients, $a_{l j}$ and $b_{l k}(l=1,2, \ldots, 6 ; j=1,2 ; k=$ $1,2,3$ ), are long expressions involving: (i) components of Green's tensor and their first and second order derivatives with respect to Cartesian coordinates, and (ii) various correlation functions (there are $49+2$ in all, the last two being the auto-correlation function of the non-generalized thermomechanical coupling parameter and the cross-correlation function between the two thermo-mechanical coupling parameters) and their first and second order derivatives with respect to Cartesian coordinates.

\section{Transformation to radial forms}

The transformation of these integrals from Cartesian to radial forms are therefore carried out under the substitutions

$$
\vec{r}=\vec{x}-\vec{x}^{\prime}=(\xi, \eta, \zeta)=(r \cos \theta, r \sin \theta \cos \phi, r \sin \theta \sin \phi)
$$

so that

$$
\frac{\partial}{\partial x}=\frac{\partial}{\partial \xi}=-\frac{\partial}{\partial x^{\prime}}, \quad \text { etc. }
$$

The Green's tensor and the correlation functions are all functions of $r=$ $|\vec{r}|$ alone. Thus 30 coefficients $a_{l j}$ and $b_{l k}$ are all functions of the wave number $k$ alone. The expressions for $a_{11}, a_{22}, a_{62}, b_{23}, b_{51}, b_{63}$, which enter into the dispersion relations governing wave propagation in the medium are transformed into radial forms; the other coefficients which involve similar lengthy and cumbersome expressions are omitted. The expression for $a_{11}$ dependent on thermal parameters is computed as 


$$
\begin{aligned}
a_{11}= & 4 \pi k \int_{0}^{\infty} r^{2}\left[\left\{k R_{1}-\frac{1}{k} \omega^{2}\left(R_{\lambda \rho}+R_{\mu \rho}\right)\right\}\left\{f^{\prime}\left(G_{1}\right)_{11}^{\prime \prime}+\frac{\left(G_{1}\right)_{11}^{\prime}}{r}\left(f+f^{\prime \prime}\right)\right\}-R_{1}^{\prime}\left\{f^{\prime \prime \prime}\left(G_{1}\right)_{11}^{\prime \prime}\right.\right. \\
& \left.\left.-\frac{1}{r}\left(G_{1}\right)_{11}^{\prime}\left(f^{\prime}+f^{\prime \prime \prime}\right)\right\}+\frac{1}{2}\left(R_{\lambda \lambda}^{\prime}+R_{\mu \lambda}^{\prime}\right)\left\{G_{1}\right)_{11}^{\prime \prime}+\left(G_{1}\right)_{33}^{\prime \prime}-\frac{1}{r}\left(\left(G_{1}\right)_{11}^{\prime}+\left(G_{1}\right)_{33}^{\prime}\right)\right\}\left(f^{\prime}+f^{\prime \prime \prime}\right) \\
& +\left(\left(G_{1}\right)_{11}^{\prime \prime}+2\left(G_{1}\right)_{11}^{\prime}\right)\left[\left\{\frac{1}{k} \omega^{2}\left(R_{\mu \rho}+R_{\mathrm{kp}}\right)-k R_{2}\right\} f+\frac{1}{k} R_{2}^{\prime} f^{\prime}\right]+\left(G_{1}\right)_{11}^{\prime}\left[k \left\{\left(R_{1}^{\prime}+R_{2}^{\prime}\right) f^{\prime \prime}+\right.\right. \\
& \left.\left.R_{2}^{\prime}\left(f+f^{\prime \prime}\right)\right\}-R_{\lambda \lambda}^{\prime \prime} f^{\prime \prime \prime}-\frac{1}{r} R_{\lambda \lambda}^{\prime}\left(f^{\prime}+f^{\prime \prime \prime}\right)\right]+\frac{1}{2}\left[\left(R_{\lambda \lambda}^{\prime \prime}-\frac{1}{2} R_{\lambda \lambda}^{\prime}\right)\left(\left(G_{1}\right)_{11}^{\prime}+\left(G_{1}\right)_{33}^{\prime}\right)+\left(R_{\mu \lambda}^{\prime \prime}-\frac{1}{r} R_{\mu \lambda}^{\prime}\right)\right. \\
& \left\{\left(G_{1}\right)_{33}^{\prime} f^{\prime}+\left(f^{\prime}+f^{\prime \prime \prime \prime}\right)\left(G_{1}\right)_{11\}}^{\prime}\right]+\frac{1}{r} R_{\mu \lambda}^{\prime} f^{\prime}\left(\left(G_{1}\right)_{11}^{\prime}+\left(G_{1}\right)_{33}^{\prime}\right)+\left(R_{2}^{\prime \prime}-\frac{1}{r} R_{2}^{\prime}\right)\left(G_{1}\right)_{11}^{\prime}\left(f^{\prime}+f^{\prime \prime \prime}\right) \\
& -\omega^{2}\left[\frac{1}{k}\left(G_{1}\right)_{11}^{\prime}\left\{f^{\prime \prime}\left(R_{\lambda \rho}^{\prime}+2 R_{\mu \rho}^{\prime}+R_{\mathrm{kp}}^{\prime}\right)+\left(R_{\mu \rho}+R_{k \rho}\right)\left(f+f^{\prime \prime}\right)\right\}-\left(G_{1}\right)_{11}\left\{\frac{1}{k} f \omega^{2} R_{\rho \rho}\right.\right. \\
& \left.\left.-k f\left(R_{\rho \lambda}+2 R_{\rho \mu}+R_{\rho \kappa}\right)+f^{\prime}\left(R_{\rho \lambda}^{\prime}+2 R_{\rho \mu}^{\prime}+R_{\rho \kappa}^{\prime}\right)\right\}\right] d r \\
- & 4 i \pi k \omega \theta_{0}\left(1-i \delta_{l k} t_{0} \omega k\right) \int_{0}^{\infty}\left[\left(R_{m m}-i \omega R_{m m} .\right) G_{4}^{\prime}+\left(R_{m m}^{\prime}-i \omega R_{m m}^{\prime} .\right) G_{4}\right] r^{2} f^{\prime} d r .
\end{aligned}
$$

where

$$
\begin{gathered}
R_{1}(r)=\left\langle\left(\lambda_{1}+\mu_{1}\right)\left(\lambda_{1}^{\prime}+2 \mu_{1}^{\prime}+\kappa_{1}^{\prime}\right)\right\rangle, \\
R_{2}(r)=\left\langle\left(\mu_{1}+\kappa_{1}\right)\left(\lambda_{1}^{\prime}+2 \mu_{1}^{\prime}+\kappa_{1}^{\prime}\right)\right\rangle, \\
R_{\lambda \rho}(r)=\left\langle\lambda_{1} \rho_{1}^{\prime}\right\rangle, R_{m m}(r)=\left\langle m m^{\prime}\right\rangle, R_{m m} \bullet(r)=\left\langle m m^{\bullet}\right\rangle, \text { etc. } \\
\lambda_{1}=\lambda_{1}(\vec{x}), \lambda_{1}^{\prime}=\lambda_{1}^{\prime}\left(\vec{x}^{\prime}\right), \\
m_{1}=m_{1}(\vec{x}), m_{1}^{\bullet}=m_{1} t=m_{1}^{\bullet}(\vec{x}), \\
m_{1}^{\prime}=m_{1}\left(\vec{x}^{\prime}\right), m_{1}^{\bullet \prime}=m_{1} t=m_{1}^{\bullet}\left(\vec{x}^{\prime}\right), \text { etc. }
\end{gathered}
$$

and

$$
\begin{gathered}
f=\frac{\sin k r}{k r}, f^{\prime}=\frac{d f}{d(k r)} \\
\left(G_{1}\right)_{11}^{\prime}=\frac{d\left(G_{1}\right)_{11}}{d r}, \quad R_{1}^{\prime}=\frac{d R_{1}}{d r}, \text { etc. } \\
R_{7}(r)=\left\langle\left(\alpha_{1}+\beta_{1}\right)\left(2 \kappa_{1}^{\prime}-j \omega^{2} \rho_{1}^{\prime}\right)\right\rangle .
\end{gathered}
$$

The radial expressions for coefficients $a_{22}, a_{62}, b_{23}, b_{51}, b_{63}$, which are independent of thermal field can be found in [18]. However the expression for $b_{63}$ is reproduced illustratively as also as a ready reference for the analysis given below. 


$$
\begin{aligned}
& b_{63}=4 \pi k \int_{0}^{\infty} r^{2}\left[\left\{R_{\mathrm{KK}} f^{\prime}+\frac{1}{k} R_{\mathrm{KK}}^{\prime} f^{\prime \prime}+\frac{1}{2 k} R_{\mathrm{K} \kappa}^{\prime}\left(f+f^{\prime}\right)\right\}\left(G_{1}\right)_{11}^{\prime}-\frac{1}{2 k}\left\{k^{2}\left(R_{\alpha \gamma}+R_{\beta \gamma}\right)+R_{7}\right\}\right. \\
& \left\{\frac{2}{r} f\left(G_{4}\right)_{33}^{\prime}+\left(f+f^{\prime \prime}\right)\left(\frac{1}{r}\left(G_{4}\right)_{33}^{\prime}-\left(G_{4}\right)_{33}^{\prime \prime}\right)\right\}+\frac{1}{2}\left(R_{\alpha \beta}^{\prime}+R_{\beta \beta}^{\prime}\right)\left(\left(G_{4}\right)_{11}^{\prime \prime}-\left(G_{4}\right)_{11}^{\prime}\right) \\
& \left(f^{\prime}+f^{\prime \prime}\right)+\left(R_{x y}^{\prime}+R_{p y}^{\prime}\right)\left\{\frac{1}{r} f^{\prime}\left(G_{4}\right)_{33}^{\prime}+\frac{1}{2}\left(f^{\prime}+f^{\prime \prime}\right)\left(\left(G_{4}\right)_{33}^{\prime \prime}-\frac{1}{r}\left(G_{4}\right)_{33}^{\prime}\right)\right\}+\frac{1}{2 k}\left(k^{2} R_{u \gamma}^{\prime}\right. \\
& \left.+2 R_{\mathrm{\alpha K}}^{\prime}-j \omega^{2} R_{\alpha \rho}\right)\left(f+f^{\prime \prime}\right)\left(G_{4}\right)_{3 \xi}^{\prime}+\left\{\frac{1}{2}\left(R_{\alpha \beta}^{\prime \prime}-R_{\alpha \beta}^{\prime}\right)\left(f^{\prime}+f^{\prime \prime \prime}\right)+R_{\alpha \beta}^{\prime} f^{\prime}\right\}\left(G_{4}\right)_{11}^{\prime} \\
& +\frac{1}{2}\left(R_{z}^{\prime \prime}-R_{z}^{\prime}\right)\left(f^{\prime}+f^{\prime \prime \prime}\right)\left(G_{4}\right)_{3,3}^{\prime}+\left\{R_{v y}^{\prime} f^{\prime}-\frac{1}{k}\left(R_{\gamma y} k^{2}+2 R_{\gamma k}-j \omega^{2} R_{p \rho}\right) f\right\}\left\{\left(G_{4}\right)_{33}^{\prime \prime}+\frac{2}{r}\left(G_{4}\right)_{3,3}^{\prime}\right\} \\
& \left.+\frac{1}{2 k}\left(G_{4}\right)_{33}^{\prime}\left[f^{\prime \prime}\left\{R_{Q}+k^{2}\left(R_{\beta \gamma}^{\prime}+4 R_{\gamma \gamma}^{\prime}\right)\right\}+\left\{k^{2}\left(R_{\beta \gamma}^{\prime}+2 R_{\gamma \gamma}^{\prime}\right)+R_{10}\right\}\right)\right]+\frac{1}{2}\left(R_{\beta \beta}^{\prime \prime}-\frac{1}{r} R_{\beta \beta}^{\prime}\right)
\end{aligned}
$$



$$
\begin{aligned}
& \left.\times f\left(G_{4}\right)_{33}-\left(G_{4}\right)_{33}^{\prime} f^{\prime}\left(2 R_{\mathrm{k} \beta}^{\prime}-j \omega^{2} R_{\rho \beta}^{\prime}\right)\right] d r
\end{aligned}
$$

where

$$
\begin{aligned}
& R_{7}(r)=\left\langle\left(\alpha_{1}+\beta_{1}\right)\left(2 \kappa_{1}^{\prime}-j \omega^{2} \rho_{1}^{\prime}\right)\right\rangle, \\
& R_{8}(r)=\left\langle\left(\alpha_{1}+\beta_{1}+\gamma_{1}\right) \gamma_{1}^{\prime}\right\rangle, \\
& R_{9}(r)=\left\langle\left(\beta_{1}+4 \gamma_{1}\right)\left(2 \kappa_{1}^{\prime}-j \omega^{2} \rho_{1}^{\prime}\right)\right\rangle, \\
& R_{10}(r)=\left\langle\left(\beta_{1}+2 \gamma_{1}\right)\left(2 \kappa_{1}^{\prime}-j \omega^{2} \rho_{1}^{\prime}\right)\right\rangle, \\
& R_{11}(r)=\left\langle\left(2 \kappa_{1}-j \omega^{2} \rho_{1}\right)\left(2 \kappa_{1}^{\prime}-j \omega^{2} \rho_{1}^{\prime}\right)\right\rangle= \\
& \quad=4\left\langle\kappa_{1} \kappa_{1}^{\prime}\right\rangle-2 j \omega^{2}\left[\left\langle\rho_{1} \kappa_{1}^{\prime}\right\rangle+\left\langle\rho_{1}^{\prime} \kappa_{1}^{\prime}\right\rangle\right]+j^{2} \omega^{4}\left\langle\rho_{1} \rho_{1}^{\prime}\right\rangle .
\end{aligned}
$$

\section{Analysis of equations (51)-(56)}

(i) Let

$$
k^{2}=k_{l}^{2}+\varepsilon^{2} \delta_{l}
$$

where

$$
k_{l}^{2}=\frac{\rho_{0} \omega^{2}}{\alpha_{0}+\beta_{0}+\gamma_{0}}
$$

be taken as a solution of Eqs. (51)-(56). Substituting in (54) one gets the equation

$$
\begin{aligned}
& \left\{-\left[2 \kappa_{0}-\rho_{0} j \omega^{2}+\left(\alpha_{0}+\beta_{0}+\gamma_{0}\right)\right] k_{l}^{2}\right\} B_{1} \\
& -\varepsilon^{2}\left\{\left[a_{41} A_{1}+a_{42} A_{2}+\left[b_{41}+\left(\alpha_{0}+\beta_{0}+\gamma_{0}\right) \delta_{l}\right] B_{1}+\right.\right. \\
& \left.\left.b_{42} B_{2}+b_{43} B_{3}\right]_{k=k_{l}}\right\}=0 .
\end{aligned}
$$


Hence

$$
B_{1} \approx O\left(\varepsilon^{2}\right)
$$

Similarly it can be shown from Eq. (57) that

$$
B_{2} \approx O\left(\varepsilon^{2}\right)
$$

From (52) one gets

$$
\begin{aligned}
& {\left[\rho_{0} \omega^{2}-\left(\mu_{0}+\kappa_{0}\right) k_{l}^{2}\right] A_{2}-i \kappa_{0} k_{l} B_{3}} \\
& -\varepsilon^{2}\left[a_{21} A_{1}+\left\{a_{22}+\left(\mu_{0}+\kappa_{0}\right) \delta_{l}\right\} A_{2}+b_{21} B_{1}+b_{22} B_{2}+\right. \\
& \left.\left\{b_{23}-\frac{i \kappa_{0}}{2 k_{l}}\right\} B_{3}\right]_{k=k_{l}}=0 .
\end{aligned}
$$

Similarly from (56) one gets

$$
\begin{aligned}
& i \kappa_{0} k_{l} A_{2}+\left(\rho_{0} j \omega^{2}-2 \kappa_{0}-\gamma_{0} k_{l}^{2}\right) B_{3} \\
& -\varepsilon^{2}\left[a_{61} A_{1}+\left(a_{62}+\frac{i \kappa_{0}}{2 k_{l}}\right) A_{2}+b_{61} B_{1}+b_{62} B_{2}+\left(\gamma_{0} \delta_{l}+b_{63}\right) B_{3}\right]_{k=k_{l}}=0 .
\end{aligned}
$$

Thus from these two equations it can be concluded that for finite $A_{1}$

$$
A_{2} \approx O\left(\varepsilon^{2}\right) \text { and } B_{3} \approx O\left(\varepsilon^{2}\right)
$$

Lastly the first Eq. (51) becomes, on neglecting $A_{2}, B_{1}, B_{2}$, and $B_{3}$, as these amplitudes are small to the order $\varepsilon^{2}$ only:

$$
\begin{aligned}
& {\left[\rho_{0} \omega^{2}-\left(\lambda_{0}+2 \mu_{0}+\kappa_{0}\right)\left(k_{l}^{2}+\varepsilon^{2} \delta_{l}\right)\right] A_{1}} \\
& -\varepsilon^{2} \frac{\omega \theta_{0}\left(1-i \omega t_{0} \delta_{l k}\right) m_{2}\left(i m_{2}+\omega m_{3}\right) k_{l}^{2}}{i \omega\left(1-i t_{0} \omega\right) \eta_{0}-\nu_{0} k_{l}^{2}} A_{1} \\
& -\varepsilon^{2} \omega \theta_{0}\left(1-i \omega t_{0} \delta_{l k}\right) m_{2}\left(i m_{2}+\omega m_{3}\right) k_{l} A_{1} \int_{0}^{\propto} e^{i \beta r} \sin k_{l} r d r \\
& -\varepsilon^{2}\left[a_{11}\right]_{k=k_{l}} A_{1}=0 .
\end{aligned}
$$

Rewriting, one obtains, assuming $A_{1}$ to be finite,

$$
-\left(\lambda_{0}+2 \mu_{0}+\kappa_{0}\right) \varepsilon^{2} \delta_{l} A_{1}+\varepsilon^{2} D_{3} A_{1}+\varepsilon^{2} D_{4} A_{1}-\varepsilon^{2} a_{11}\left(k_{l}\right) A_{1}=0,
$$

where

$$
\begin{aligned}
& D_{3}\left(k_{l}\right)=-\frac{\omega \theta_{0}\left(1-i \omega t_{0} \delta_{l k}\right) m_{2}\left(i m_{2}+\omega m_{3}\right) k^{2}}{i \omega\left(1-i t_{0} \omega\right) \eta_{0}-\nu_{0} k^{2}} \\
& D_{4}\left(k_{l}\right)=-\omega \theta_{0}\left(1-i \omega t_{0} \delta_{l k}\right) m_{2}\left(i m_{2}+\omega m_{3}\right) k_{l} \int_{0}^{\propto} e^{i \beta r} \sin k_{l} r d r .
\end{aligned}
$$


Hence

$$
\delta_{l}=\frac{D_{3}\left(k_{l}\right)+D_{4}\left(k_{l}\right)-a_{11}\left(k_{l}\right)}{\lambda_{0}+2 \mu_{0}+\kappa_{0}} .
$$

where

$$
\begin{aligned}
& D_{3}\left(k_{l}\right)=-\frac{\omega \theta_{0}\left(1-i \omega t_{0} \delta_{l k}\right) m_{2}\left(i m_{2}+\omega m_{3}\right) k^{2}}{i \omega\left(1-i t_{0} \omega\right) \eta_{0}-\nu_{0} k^{2}} \\
& =\frac{-\omega \theta_{0} m_{2}^{2} k^{2}\left(1-i t_{0} \omega\right)\left[\left(\omega \eta_{0}-\nu_{0} k^{2}\right)+i t_{0} \omega \eta_{0}\right]}{\left(\omega \eta_{0}-\nu_{0} k^{2}\right)^{2}+\left(t_{0} \omega \eta_{0}\right)^{2}}, t_{1}=0, \delta_{l k}=1,(L-S), \\
& =\frac{-\omega \theta_{0} m_{2} k^{2}\left[\left(m_{2}-i \omega m_{3}\right)\left(\omega \eta_{0}-\nu_{0} k^{2}\right)+i t_{0} \omega \eta_{0}\right]}{\left(\omega \eta_{0}-\nu_{0} k^{2}\right)^{2}+\left(t_{0} \omega \eta_{0}\right)^{2}}, \delta_{l k}=0, t_{1}>1,(G-L) .
\end{aligned}
$$

and

$$
\begin{aligned}
& D_{4}\left(k_{l}\right)=-\omega \theta_{0}\left(1-i \omega t_{0} \delta_{l k}\right) m_{2}\left(i m_{2}+\omega m_{3}\right) k_{l} \int_{0}^{\infty} e^{i \beta r} \sin k_{l} r d r \\
& =-\omega \theta_{0} m_{2}^{2} k_{l}\left[t_{0} \omega+i\right] \int_{0}^{\infty} e^{i \beta r} \sin k_{l} r d r, t_{1}=0, \delta_{l k}=1,(L-S), \\
& =-\omega \theta_{0} m_{2} k_{l}\left[\omega m_{3}+i m_{2}\right] \int_{0}^{\infty} e^{i \beta r} \sin k_{l} r d r, t_{1}>0, \delta_{l k}=0,(G-L) .
\end{aligned}
$$

Thus Eq. (68) determines $\delta_{l}$ given by (63). The integral in $D_{4}$ can be easily evaluated. The change in wave number $\delta_{l}$ which is small therefore depends upon the random variation of the generalized thermal field and measured in terms of statistical means, $m_{2}, m_{3}$, for both L-S and G-L theories. For L-S, the change depends upon $m_{2}$ only, and for G-L upon $m_{2}, m_{3}$, (for, $\mathrm{L}-\mathrm{S}, t_{1}=0, \delta_{l k}=1, k=1$, and for G-L, $t_{1}>0, \delta_{l k}=0, k=2$. Generalized thermal relaxation times $t_{0}$ and $t_{1}$ satisfy $([10,21,29,42])$ the inequalities $\left.t_{1} \geq t_{0} \geq 0\right)$.

Thus it is observed that for waves for which the wave number $k$ satisfies Eqs. (63)-(64), the amplitude $A_{1}$ is important in comparison to other amplitudes $A_{2}, B_{1}, B_{2}$, and $B_{3}$. Therefore Eq. (63), which represents longitudinal type of displacement waves, is modified due to random fluctuation of inhomogeneities of the medium such that the wave number is increased by $\varepsilon^{2} \frac{\delta_{l}}{2 k_{l}}$.

(ii) Next let us consider waves for which

$$
k^{2}=k_{n}^{2}+\varepsilon^{2} \delta_{n}, \quad \text { where } k_{n}^{2}=\frac{\rho_{0} j \omega^{2}-2 \kappa_{0}}{\alpha_{0}+\beta_{0}+\gamma_{0}} .
$$


From (51) one gets

$$
\begin{aligned}
& {\left[\rho_{0} \omega^{2}-\left(\lambda_{0}+2 \mu_{0}+\kappa_{0}\right) k_{n}^{2}\right] A_{1}-\varepsilon^{2}\left(\lambda_{0}+2 \mu_{0}+\kappa_{0}\right) \delta_{n} A_{1}} \\
& -\varepsilon^{2} \frac{\omega \theta_{0}\left(1-i \omega t_{0} \delta_{l k}\right) m_{2}\left(i m_{2}+\omega m_{3}\right) k_{n}^{2}}{i \omega\left(1-i t_{0} \omega\right) \eta_{0}-\nu_{0} k^{2}} A_{1} \\
& -\varepsilon^{2} \omega \theta_{0}\left(1-i \omega t_{0} \delta_{l k}\right) m_{2}\left(i m_{2}+\omega m_{3}\right) k_{n} A_{1} \int_{0}^{\propto} e^{i \beta r} \sin k_{n} r d r \\
& -\varepsilon^{2}\left[a_{11} A_{1}+a_{12} A_{2}+b_{11} B_{1}+b_{12} B_{2}+b_{13} B_{3}\right]_{k=k_{n}}=0 .
\end{aligned}
$$

This shows that

$$
A_{1} \approx O\left(\varepsilon^{2}\right)
$$

From (53) it follows that

$$
\left[i \kappa_{0}+\frac{\varepsilon^{2} \delta_{n}}{2 k_{n}^{2}} i \kappa_{0}\right] k_{n} B_{2}-\varepsilon^{2}\left[a_{31} A_{1}+a_{32} A_{2}+b_{31} B_{1}+b_{32} B_{2}+b_{33} B_{3}\right]=0
$$

This shows that

$$
B_{2} \approx O\left(\varepsilon^{2}\right) .
$$

Also from (52) and (56) it can be shown that for finite $B_{1}$,

$$
\begin{aligned}
& {\left[\rho_{0} \omega^{2}-\left(\mu_{0}+\kappa_{0}\right) k_{n}^{2}\right] A_{2}-i \kappa_{0} k_{n} B_{3}-\varepsilon^{2} \delta_{n}\left(\mu_{0}+\kappa_{0}\right) A_{2}-\frac{\varepsilon^{2} \delta_{n}}{2 k_{n}^{2}} i \kappa_{0} k_{n} B_{3}} \\
& -\varepsilon^{2}\left[a_{21} A_{1}+a_{22} A_{2}+b_{21} B_{1}+b_{22} B_{2}+b_{23} B_{3}\right]=0
\end{aligned}
$$

and

$$
\begin{aligned}
& i \kappa_{0} k_{n} A_{2}+\left(\rho_{0} j \omega^{2}-2 \kappa_{0}-\gamma_{0} k_{n}^{2}\right) B_{3}+\frac{\varepsilon^{2} \delta_{n}}{2 k_{n}^{2}} i \kappa_{0} k_{n} A_{2}-\varepsilon^{2} \delta_{n} \gamma_{0} B_{3} \\
& -\varepsilon^{2} \int\left[a_{61} A_{1}+a_{62} A_{2}+b_{61} B_{1}+b_{62} B_{2}+b_{63} B_{3}\right]=0,
\end{aligned}
$$

respectively. From these two Eqs. (73a) and (73b) it is clear that

$$
A_{2} \approx O\left(\varepsilon^{2}\right) \text { and } B_{3} \approx O\left(\varepsilon^{2}\right) .
$$

Equation (54) however, determines $\delta_{n}$, since

$$
\begin{aligned}
& -\varepsilon^{2} \delta_{n}\left(\alpha_{0}+\beta_{0}+\gamma_{0}\right) B_{1} \\
& -\varepsilon^{2} \int\left[a_{41} A_{1}+a_{42} A_{2}+b_{41} B_{1}+b_{42} B_{2}+b_{43} B_{3}\right]=0
\end{aligned}
$$

and $\left(A_{1}, A_{2}, B_{2}, B_{3}\right) \approx O\left(\varepsilon^{2}\right)$. Hence, if $B_{1}$ is finite, then

$$
\delta_{n}=\frac{-b_{41}}{\alpha_{0}+\beta_{0}+\gamma_{0}} .
$$


Therefore, $\delta_{n}=0$ if $b_{41}=0$ and amplitude $B_{1}$ is finite.

It is observed that for the waves represented by (71), the amplitude $B_{1}$ is important in comparison to the amplitudes $A_{1}, A_{2}, B_{2}$, and $B_{3}$. These are amplitudes of longitudinal type microrotation waves propagating in the random generalized thermoelastic micropolar field but are scarcely affected by the random inhomogeneities of the medium.

(iii) Next let us examine the propagation of elastic waves for which

$$
k^{2}=k_{c}^{2}+\varepsilon^{2} \delta_{c}, \quad k_{c}^{2}=\frac{\rho_{0} \omega^{2}}{\lambda_{0}+2 \mu_{0}},
$$

and

$$
k^{2}=k_{s}^{2}+\varepsilon^{2} \delta_{s}, k_{s}^{2}=\frac{\rho_{0} \omega^{2}}{\mu_{0}} .
$$

Substituting (76) in (51) one observes

$$
\begin{aligned}
& {\left[-\kappa_{0} \rho_{0} \omega^{2}-\varepsilon^{2}\left(\lambda_{0}+2 \mu_{0}+\kappa_{0}\right)\left(\lambda_{0}+2 \mu_{0}\right) \delta_{c}\right] A_{1}} \\
& -\varepsilon^{2} \frac{\omega \theta_{0}\left(1-i \omega t_{0} \delta_{l k}\right) m_{2}\left(i m_{2}+\omega m_{3}\right) k_{c}^{2}}{i \omega\left(1-i t_{0} \omega\right) \eta_{0}-\nu_{o} k_{c}^{2}}\left(\lambda_{0}+2 \mu_{0}\right) A_{1} \\
& -\varepsilon^{2} \omega \theta_{0}\left(1-i \omega t_{0} \delta_{l k}\right) m_{2}\left(i m_{2}+\omega m_{3}\right)\left(\lambda_{0}+2 \mu_{0}\right) k_{c} A_{1} \int_{0}^{\alpha} e^{i \beta r} \sin k r d r \\
& -\varepsilon^{2}\left(a_{11} A_{1}+a_{12} A_{2}+b_{11} B_{1}+b_{12} B_{2}+b_{13} B_{3}\right)\left(\lambda_{0}+2 \mu_{0}\right)=0 .
\end{aligned}
$$

Hence

$$
A_{1} \approx O\left(\varepsilon^{2}\right) \text {. }
$$

Substituting (76) in (55) it can be similarly shown that

$$
B_{2} \approx O\left(\varepsilon^{2}\right) \text {. }
$$

From (54) one can similarly conclude that

$$
B_{1} \approx O\left(\varepsilon^{2}\right) .
$$

Two equations (52) and (56) are coupled with the transverse amplitudes $A_{2}$ and $B_{3}$ such that

$$
\left[\rho_{0} \omega^{2}-\left(\mu_{0}+\kappa_{0}\right) k^{2}\right] A_{2}-i \kappa_{0} k B_{3}-\varepsilon^{2}\left(b_{22} B_{2}+b_{23} B_{3}\right)=0
$$

and

$$
i \kappa_{0} k A_{2}+\left(\rho_{0} j \omega^{2}-2 \kappa_{0}-\gamma_{0} k^{2}\right) B_{3}-\varepsilon^{2}\left(b_{62} B_{2}+b_{63} B_{3}\right)=0 .
$$


Eliminating $A_{2}$ and $B_{3}$ one obtains the dispersion equation for two sets of transverse type of displacement and microrotation waves propagating in the uncoupled micropolar elastic medium and independent of thermal field, as no thermal parameter is involved in any of the integrals $b_{22}, b_{23}, b_{62}$, and $b_{63}$, given by

$$
\begin{aligned}
& {\left[\rho_{0} \omega^{2}-\left(\mu_{0}+\kappa_{0}\right) k^{2}\right]\left(\rho_{0} j \omega^{2}-2 \kappa_{0}-\gamma_{0} k^{2}\right)-\kappa_{0}^{2} k^{2}} \\
& -\varepsilon^{2}\left[a_{22}\left(\rho_{0} j \omega^{2}-2 \kappa_{0}-\gamma_{0} k^{2}\right)+b_{63}\left[\rho_{0} \omega^{2}-\left(\mu_{0}+\kappa_{0}\right) k^{2}\right]\right. \\
& \left.+i \kappa_{0} k\left(a_{62}-b_{23}\right)\right]=0 .
\end{aligned}
$$

This equation involves integrals $a_{22}, a_{62}, b_{23}$, and $b_{63}$ each involving correlation functions between non-thermal medium-parameters only. Substituting (76) in (80) and neglecting terms to the order $\varepsilon^{2}$, the deviation $\delta_{c}$ can be computed from:

$$
\begin{aligned}
& {\left[\rho_{0} \omega^{2}-\left(\mu_{0}+\kappa_{0}\right) k_{c}^{2}\right]\left(\rho_{0} j \omega^{2}-2 \kappa_{0}-\gamma_{0} k_{c}^{2}\right)-\kappa_{0}^{2} k_{c}^{2}} \\
& -\varepsilon^{2} \gamma_{0} \delta_{c}\left[\rho_{0} \omega^{2}-\left(\mu_{0}+\kappa_{0}\right) k_{c}^{2}\right]-\varepsilon^{2}\left(\mu_{0}+\kappa_{0}\right) \delta_{c}\left(\rho_{0} j \omega^{2}-2 \kappa_{0}-\gamma_{0} k_{c}^{2}\right)-\varepsilon^{2} \kappa_{0}^{2} \delta_{c} \\
& -\varepsilon^{2}\left[a_{22}\left(\rho_{0} j \omega^{2}-2 \kappa_{0}-\gamma_{0} k_{c}^{2}\right)+b_{63}\left[\rho_{0} \omega^{2}-\left(\mu_{0}+\kappa_{0}\right) k_{c}^{2}\right]\right. \\
& \left.+i \kappa_{0}\left(a_{62}-b_{23}\right) k_{c}\right]=0 .
\end{aligned}
$$

If $\varepsilon^{2}=0$, then we know

$$
\left[\rho_{0} \omega^{2}-\left(\mu_{0}+\kappa_{0}\right) k_{c}^{2}\right]\left(\rho_{0} j \omega^{2}-2 \kappa_{0}-\gamma_{0} k_{c}^{2}\right)-\kappa_{0}^{2} k_{c}^{2}=0 .
$$

Hence $\delta_{c}$ and similarly $\delta_{s}$ are obtained in the forms:

$\delta_{c} \approx-\frac{b_{63}\left[\rho_{0} \omega^{2}-\left(\mu_{0}+\kappa_{0}\right) k_{c}^{2}\right]+a_{22}\left(\rho_{0} j \omega^{2}-2 \kappa_{0}-\gamma_{0} k_{c}^{2}\right)+\left(a_{62}-b_{23}\right) i \kappa_{0} k_{c}}{\gamma_{0}\left[\rho_{0} \omega^{2}-\left(\mu_{0}+\kappa_{0}\right) k_{c}^{2}\right]+\left(\mu_{0}+\kappa_{0}\right)\left(\rho_{0} j \omega^{2}-2 \kappa_{0}-\gamma_{0} k_{c}^{2}\right)+\kappa_{0}^{2}}$,

and

$\delta_{s} \approx-\frac{b_{63}\left[\rho_{0} \omega^{2}-\left(\mu_{0}+\kappa_{0}\right) k_{s}^{2}\right]+a_{22}\left(\rho_{0} j \omega^{2}-2 \kappa_{0}-\gamma_{0} k_{s}^{2}\right)+\left(a_{62}-b_{23}\right) i \kappa_{0} k_{s}}{\gamma_{0}\left[\rho_{0} \omega^{2}-\left(\mu_{0}+\kappa_{0}\right) k_{s}^{2}\right]+\left(\mu_{0}+\kappa_{0}\right)\left(\rho_{0} j \omega^{2}-2 \kappa_{0}-\gamma_{0} k_{s}^{2}\right)+\kappa_{0}^{2}}$.

Both $\delta_{c}$ and $\delta_{s}$ are complex quantities. Computing real and imaginary parts one can write:

$$
k=k_{c}+\frac{\varepsilon^{2} \delta_{c}}{2 k_{c}}=k_{c}+\varepsilon^{2} \frac{\operatorname{Re} \delta_{c}}{2 k_{c}}+i \varepsilon^{2} \frac{\operatorname{Im} \delta_{c}}{2 k_{c}},
$$


and

$$
k=k_{s}+\frac{\varepsilon^{2} \delta_{s}}{2 k_{s}}=k_{c}+\varepsilon^{2} \frac{\operatorname{Re} \delta_{s}}{2 k_{s}}+i \varepsilon^{2} \frac{\operatorname{Im} \delta_{s}}{2 k_{s}} .
$$

Hence for longitudinal waves

$$
i \vec{k} \vec{x}=i k x=i x\left[k_{c}+\frac{\varepsilon^{2} \delta_{c}}{2 k_{c}}\right]=i x\left[k_{c}+\varepsilon^{2} \frac{\operatorname{Re} \delta_{c}}{2 k_{c}}+i \varepsilon^{2} \frac{\operatorname{Im} \delta_{c}}{2 k_{c}}\right],
$$

and for transverse waves

$$
i \vec{k} \vec{x}=i k x=i x\left[k_{s}+\frac{\varepsilon^{2} \delta_{s}}{2 k_{s}}\right]=i x\left[k_{s}+\varepsilon^{2} \frac{\operatorname{Re} \delta_{s}}{2 k_{s}}+i \varepsilon^{2} \frac{\operatorname{Im} \delta_{s}}{2 k_{s}}\right] .
$$

Thus change in the wave number and attenuation occurs in both cases, having attenuation factors of the forms

$$
\exp \left[-\frac{\varepsilon^{2} x}{2} \operatorname{Im}\left(\frac{\delta_{c}}{k_{c}}, \frac{\delta_{s}}{k_{s}}\right)\right] \text {. }
$$

It may be recalled here that the only term in Eq. (36) that might contribute to some effect of thermal parameters on $\delta_{c, s}$ is

$$
\begin{aligned}
& \int\left\langle K_{1} G_{4} R_{1}^{\prime}\right\rangle\left(\vec{A} e^{i \vec{k} \cdot \vec{x}^{\prime}}\right) d \vec{x}^{\prime} \\
& =-\omega \theta_{0}\left(1-i \delta_{l k} t_{0} \omega\right)(\vec{k} \vec{A}) e^{i \vec{k} \vec{x}} \int\left\{\vec{\nabla}\left[G_{4}(r) R_{m m}(r)\right]\right. \\
& \left.-i \omega \vec{\nabla}\left[G_{4}(r) R_{m m} \cdot\right]\right\} e^{-i \vec{k}^{\prime} \cdot \vec{r}} d \vec{r} .
\end{aligned}
$$

This term clearly does not add anything to $a_{22}$ since in our case $\vec{k} \vec{A}=$ $\left(k A_{1}, 0,0\right)$. Therefore the integrals $a_{22}, a_{62}, b_{23}$ and $b_{63}$ are all independent of the thermal field. However the integral $\int\left\langle K_{1} G_{4} R_{1}^{\prime}\right\rangle\left(\vec{A} e^{i \vec{k} \cdot \vec{x}^{\prime}}\right) d \vec{x}^{\prime}$ contributes an additional term to $a_{11}$ which is

$-4 i \pi \omega \theta_{0}\left(1-i \delta_{l k} t_{0} \omega\right) k \int_{0}^{\infty}\left[\left(R_{m m}-i \omega R_{m m} \bullet\right) G_{4}^{\prime}+\left(R_{m m}^{\prime}-i \omega R_{m m}^{\prime} \bullet G_{4}\right] r^{2} f^{\prime} d r\right.$.

This term has already been included in the radial expression for $a_{11}$ in (58).

\section{Attenuation of high frequency nonthermal waves in a particular case}

This topic has already been discussed in [18]. However for the sake of continuity of discussion, the salient features are briefly enumerated below. 
It may be noted that the expressions derived for $\delta_{c}$ and $\delta_{s}$ do not depend on generalized thermal parameters. Hence the phenomenon of propagation of transverse type of displacement and microrotation waves in the random micropolar elastic medium is considered taking

$$
\left.R_{\rho \rho}(r)=\left\langle\rho_{1}^{2}\right\rangle e^{\frac{-r}{a}} \neq 0, a\right\rangle 0,
$$

and making all other auto- and cross-correlation functions vanish. (Autocorrelation functions $\left\langle\kappa_{1} \kappa_{1}^{\prime}\right\rangle,\left\langle\beta_{1} \beta_{1}^{\prime}\right\rangle,\left\langle\gamma_{1} \gamma_{1}^{\prime}\right\rangle$, signifying effects of random variation of micropolar elastic properties of the medium appear several times in $b_{63}$ making calculations unmanageably lengthy and cumbersome and hence are omitted). Then

$$
b_{63}\left(k_{c}\right)=\frac{4 j \pi \omega^{2}\left\langle\rho_{1}^{2}\right\rangle}{k_{c}} \int_{0}^{\propto .} r e^{-\frac{r}{a}}\left[G_{4}(r)\right]_{33} \sin \left(k_{c} r\right) d r
$$

and

$$
a_{22}\left(k_{c}\right)=a_{62}\left(k_{c}\right)=b_{23}\left(k_{c}\right)=0 .
$$

where $\left[G_{4}(r)\right]_{33}$ has already been evaluated [18]. Components including $\left[G_{4}(r)\right]_{33}$ of the associated Green's tensor have been reproduced in the App. III for ready-reference.

For large $\omega$ the approximate values of $k_{c}^{2}, k_{s}^{2}, k_{m}^{2}$, and $k_{n}^{2}$ were computed to show that transverse type of displacement waves attenuate. In a similar way it was shown that high frequency transverse type microrotation waves attenuate if $j>1$. Results indicate that body waves of these types attenuate due to randomness as they propagate through the medium, only in the nonthermal environment.

\section{Computation of the dispersion of the field from the mean field}

In this section we propose to give an outline of the procedure to evaluate an expression for dispersion of the field from the mean field. It is assumed that $v_{0}(\vec{x})$ and $v(\vec{x})$ represent solutions of operator equations $L_{0} v_{0}(\vec{x})=0$ and $L v(\vec{x})=0$ respectively, where $L=L_{0}+\varepsilon L_{1}+\varepsilon^{2} L_{2}+O\left(\varepsilon^{3}\right)$.

Following Karal and Keller ([23], Eq. (5)) it can be easily shown that

$$
v=v_{0}+\varepsilon L_{0}^{-1} L_{1} v_{0}+\varepsilon^{2}\left(L_{0}^{-1} L_{1} L_{0}^{1} l_{1}+L_{0}^{-1} l_{2}\right) v_{0}+O\left(\varepsilon^{2}\right) .
$$


Hence

$\bar{v}^{T}=\bar{v}_{0}^{T}+\varepsilon{\overline{\left(L_{0}^{-1} L_{1} v_{0}\right.}}^{T}+\varepsilon^{2}\left[{\overline{\left(L_{0}^{-1} L_{1} L_{0}^{-1} L_{1} v_{0}\right)}}^{T}+{\overline{\left(L_{0}^{-1} L_{2} v_{0}\right)}}^{T}\right]+O\left(\varepsilon^{3}\right)$,

where $v^{T}$ is the transpose of $v$ and $\bar{v}^{T}$ is the complex conjugate of $v^{T}$.

Therefore

$$
\begin{aligned}
\bar{v}^{T} v & \left.=\bar{v}_{0}^{T} v_{0}+\varepsilon\left[\overline{\left(L_{0}^{-1} L_{1} v_{0}\right.}\right)^{T} v_{0}+\bar{v}_{0}^{T}\left(L_{0}^{-1} L_{1} v_{0}\right)\right] \\
& +\varepsilon^{2}\left[\overline{\left(L_{0}^{-1} L_{1} v_{0}\right)}{ }^{T}\left(L_{0}^{-1} L_{1} v_{0}\right)+\left(\overline{L_{0}^{-1} L_{1} L_{0}^{-1} L_{1} v_{0}}\right)^{T} v_{0}\right. \\
& \left.+\left(\overline{L_{0}^{-1} L_{2} v_{0}}\right)^{T} v_{0}+\bar{v}_{0}^{T}\left(L_{0}^{-1} L_{1} L_{0}^{-1} L_{1} v_{0}\right)+\bar{v}_{0}^{T}\left(L_{0}^{-1} L_{2} v_{0}\right)\right]+O\left(\varepsilon^{3}\right)
\end{aligned}
$$

We take expectation of this equation taking that $\left\langle L_{1}\right\rangle=0$, so that

$$
\left\langle\bar{v}_{0}^{T}\left(L_{0}^{-1} L_{1} v_{0}\right)\right\rangle=\bar{v}_{0}^{T} L_{0}^{-1}\left\langle L_{1}\right\rangle v_{0}=0
$$

and

$$
\left\langle\left(L_{0}^{-1} L_{1} v_{0}\right)^{T} v_{0}\right\rangle=\left(L_{0}^{-1}\left\langle L_{1}\right\rangle v_{0}\right)^{T} v_{0}=0 .
$$

The condition $\left\langle L_{1}\right\rangle=0$ with the help of Eq. (23), leads to

$$
m_{2}=i \omega m_{3} \text { and } 1=i t_{0} \omega,
$$

taking $\delta_{l k}=1$ and assuming $\omega$ to be complex. Combining these two relations one gets

$$
m_{2}=i m_{3} \times \frac{1}{i t_{0}} .
$$

Hence

$$
m_{3}=t_{0} m_{2} .
$$

Therefore

$$
\begin{aligned}
\bar{v}^{T} v & =\bar{v}_{0}^{T} v_{0}+\varepsilon^{2}\left[\left\langle{\overline{\left(L_{0}^{-1} L_{1} v_{0}\right)}}^{T}\left(L_{0}^{-1} L_{1} v_{0}\right)\right\rangle+\left\langle{\overline{\left(L_{0}^{-1} L_{1} L_{0}^{-1} L_{1} v_{0}\right)}}^{T} v_{0}\right\rangle\right. \\
& +\left\langle{\overline{\left(L_{0}^{-1} L_{2} v_{0}\right)}}^{T} v_{0}\right\rangle+\left\langle\bar{v}_{0}^{T}\left(L_{0}^{-1} L_{1} L_{0}^{-1} L_{1} v_{0}\right)\right\rangle \\
& \left.+\left\langle\bar{v}_{0}^{T}\left(L_{0}^{-1} L_{2} v_{0}\right)\right\rangle\right]+O\left(\varepsilon^{3}\right) .
\end{aligned}
$$


From (89a) we obtain on taking the expectation

$$
\langle v\rangle=v_{0}+\varepsilon^{2}\left[\left\langle L_{0}^{-1} L_{1} L_{0}^{-1} L_{1} v_{0}\right\rangle+\left\langle L_{0}^{-1} L_{2} v_{0}\right\rangle\right]+O\left(\varepsilon^{3}\right) .
$$

Hence

$$
v_{0}=\langle v\rangle-\varepsilon^{2}\left[\left\langle L_{0}^{-1} L_{1} L_{0}^{-1} L_{1} v_{0}\right\rangle+\left\langle L_{0}^{-1} L_{2} v_{0}\right\rangle\right]+O\left(\varepsilon^{3}\right) .
$$

and by iteration it follows that

$$
v_{0}=\langle v\rangle-\varepsilon^{2}\left[\left\langle L_{0}^{-1} L_{1} L_{0}^{-1} L_{1}\right\rangle\langle v\rangle+\left\langle L_{0}^{-1} L_{2}\right\rangle\langle v\rangle\right]+O\left(\varepsilon^{3}\right)
$$

and

$$
\bar{v}_{0}^{T}=\left\langle\bar{v}^{T}\right\rangle-\varepsilon^{2}\left[\left\langle{\overline{\left(L_{0}^{-1} L_{1} L_{0}^{-1} L_{1}\langle v\rangle\right)}}^{T}\right\rangle+\left\langle{\overline{\left(L_{0}^{-1} L_{2}\langle v\rangle\right)}}^{T}\right\rangle\right]+O\left(\varepsilon^{3}\right)
$$

Thus

$$
\begin{aligned}
\bar{v}_{0}^{T} v_{0} & =\left\langle\bar{v}^{T}\right\rangle\langle v\rangle-\varepsilon^{2}\left[\left\langle\overline{\left(L_{0}^{-1} L_{1} L_{0}^{-1} L_{1}\langle v\rangle\right)}\right)^{T}\right\rangle\langle v\rangle+\left\langle\bar{v}^{T}\right\rangle \\
& \times\left\langle\left(L_{0}^{-1} L_{1} L_{0}^{-1} L_{1}\langle v\rangle\right)\right\rangle+\left\langle{\overline{\left(L_{0}^{-1} L_{2}\langle v\rangle\right)}}^{T}\right\rangle\langle v\rangle \\
& \left.+\left\langle\bar{v}^{T}\right\rangle\left\langle\left(L_{0}^{-1} L_{2}\langle v\rangle\right)\right\rangle\right]+O\left(\varepsilon^{3}\right)
\end{aligned}
$$

Substituting Eqs. (92)-(94) into (91) we obtain the expression

$$
\left\langle\bar{v}^{T} v\right\rangle-\left\langle\bar{v}^{T}\right\rangle\langle v\rangle=\varepsilon^{2}\left[\left\langle{\overline{\left(L_{0}^{-1} L_{1}\langle v\rangle\right)}}^{T}\left(L_{0}^{-1} L_{1}\langle v\rangle\right)\right\rangle\right]+O\left(\varepsilon^{3}\right),
$$

which is equal to sum of variances of real and imaginary parts of all the components of $v$. This relation holds only under the condition that $m_{3}=$ $t_{0} m_{2}$. Taking in general

$$
\begin{gathered}
L=\left(M_{l j}\right), \quad(l j)=1,2,3 \\
L_{0}^{-1} f=\int\left(G_{l j}\right) e^{i \vec{k} \cdot \overrightarrow{x^{\prime}}} d \overrightarrow{x^{\prime}}, \\
\langle v(\vec{x})\rangle=\left[A_{j}\right] e^{i \vec{k} \cdot \vec{x}},
\end{gathered}
$$




$$
\begin{gathered}
\vec{k}=\vec{k}_{1}+i \vec{k}_{2}, \quad k_{1}, k_{2} \equiv \text { real }, \\
\left\langle L_{1}\right\rangle=0,
\end{gathered}
$$

it has been shown that

$$
\begin{aligned}
\left\langle\bar{v}^{T} v\right\rangle-\left\langle\bar{v}^{T}\right\rangle\langle v\rangle & =\varepsilon^{2} e^{-2 \operatorname{Im} \vec{k} \cdot \vec{x}}\left(\sum\left|C_{l}\right|^{2}\right) \\
& =\varepsilon^{2} e^{-2 \operatorname{Im} \vec{k} \vec{x}} \ell\left(A_{l}\right) .
\end{aligned}
$$

where $\ell\left(A_{l}\right) \geq 0$ is seen from (92) to be a bilinear function of the amplitudes $A_{l}$. Then Eq. (100) shows that the dispersion $(\sqrt{\text { variance }})$ of the field from the mean field represented by the plane wave (98), is a small quantity of order $\varepsilon$ and that it decays as the wave propagates in those cases where $\operatorname{Im} k>0$. It follows that [18] the field may differ from the plane wave mean field by a quantity of order $\varepsilon$ with finite probability.

\section{Variance for micropolar generalized thermo- elastic wave propagation in the random medium}

This analysis is next applied to the waves in the random micropolar generalized thermoelastic medium. In this case

$$
\begin{aligned}
& \left(L_{0}^{-1} L_{1}<v \cdot>\right)^{T}=\left[e^{i \vec{k} \cdot \bar{x}}\left[\begin{array}{ccc}
G_{0} & G_{1} & 0 \\
G_{2} & G_{3} & 0 \\
0 & 0 & G_{4}
\end{array}\right]\left[\begin{array}{ccc}
M_{1} & P_{1} & K_{1} \\
N_{1} & Q_{1} & 0 \\
R_{1} & 0 & S_{1}
\end{array}\right]\left[\begin{array}{l}
\vec{A} \\
\vec{B} \\
C
\end{array}\right] e^{-i \vec{k} \cdot\left(\vec{x}-\vec{x}^{\prime}\right)}\right]^{T} d \vec{x}^{\prime} \\
& =\left[e^{i \vec{k} \cdot \vec{x}} \int\left[\begin{array}{ccc}
G_{0} & G_{1} & 0 \\
G_{2} & G_{3} & 0 \\
0 & 0 & G_{4}
\end{array}\right]\left[\begin{array}{l}
M_{1} \vec{A}+P_{1} \vec{B}+K_{1} C \\
N_{1} \vec{A}+Q_{1} \vec{B} \\
R_{1} \vec{A}+S_{1} C
\end{array}\right] e^{-i \vec{k} \cdot\left(\vec{x}-\vec{x}^{\prime}\right)}\right]^{T} d \vec{x}^{\prime} \\
& =\left[e^{i \vec{k} \cdot \vec{x}} \int\left[\begin{array}{l}
G_{0}\left(M_{1} \vec{A}+P_{1} \vec{B}+K_{1} C\right)+G_{1}\left(N_{1} \vec{A}+Q_{1} \vec{B}\right) \\
G_{2}\left(M_{1} \vec{A}+P_{1} \vec{B}+K_{1} C\right)+G_{3}\left(N_{1} \vec{A}+Q_{1} \vec{B}\right) \\
G_{4}\left(R_{1} \vec{A}+S_{1} C\right)
\end{array}\right] e^{-i \vec{k} \cdot\left(\vec{x}-\vec{x}^{\prime}\right)}\right]^{T} d \vec{x}^{\prime}
\end{aligned}
$$

where 


$$
\begin{aligned}
& M \vec{u}=\rho \frac{\partial^{2} \vec{u}}{\partial t^{2}}-(\lambda+\mu) \vec{\nabla}(\vec{\nabla} \vec{u})-(\mu+\kappa) \nabla^{2} \vec{u}-\vec{\nabla} \lambda(\vec{\nabla} \vec{u})-\vec{\nabla} \mu \times(\vec{\nabla} \times \vec{u})-(\vec{\nabla}(2 \mu+\kappa) \vec{\nabla}) \vec{u} \\
& M_{1}\left(\vec{A} e^{i \vec{k} \cdot \vec{x}}\right)=\left[-\rho_{1} \omega^{2} \vec{A}+\left(\lambda_{1}+\mu_{1}\right)(\vec{k} \vec{A}) \vec{k}+\left(\mu_{1}+\kappa_{1}\right) k^{2} \vec{A}\right. \\
& \left.-i(\vec{k} \vec{A}) \vec{\nabla} \lambda_{1}-i \vec{\nabla} \mu_{1} \times(\vec{k} \times \vec{A})-i\left(\vec{\nabla}\left(2 \mu_{1}+\kappa_{1}\right) \vec{k}\right) \vec{A}\right] e^{i \vec{k} \cdot \vec{x}} \\
& P_{1}\left(\vec{B} e^{i \vec{k} \cdot \bar{x}}\right)=-\vec{\nabla} \times\left(\kappa_{1} \vec{B} e^{i \vec{k} \cdot \vec{x}}\right)=-\left[\vec{\nabla} k_{1} \times \vec{B}+i \kappa_{1} \vec{k} \times \vec{B}\right] e^{i \vec{k} \cdot \vec{x}}, \\
& K_{1}\left(C e^{i \vec{k} \cdot \vec{x}}\right)=\left[\vec{\nabla}\left(m_{1}\right)-i \omega \vec{\nabla} m_{1}^{*}\right] C e^{i \vec{k} \cdot \vec{x}}=\left[\vec{\nabla} m_{1}+i m_{1} \vec{k}-i \omega\left(\vec{\nabla} m_{1}^{*}+i m_{1}^{*}\right)\right] C e^{i \vec{k} \cdot \vec{x}}, \\
& N_{1}\left(\vec{A} e^{i \vec{k} \cdot \vec{x}}\right)=-\kappa_{1}(\vec{\nabla} \times)\left(\vec{A} e^{i \vec{k} \cdot \vec{x}}\right)=-i \kappa_{1}(\vec{k} \times \vec{A}) e^{i \vec{k} \cdot \vec{x}} \\
& Q_{1}\left(\vec{B} e^{i \vec{k} \cdot \vec{k}}\right)=\left[\left(-\rho_{1} j \omega^{2}+2 \kappa_{1}\right) \vec{B}+\left(\alpha_{1}+\beta_{1}\right)(\vec{k} \cdot \vec{B}) \vec{k}-i(\vec{k} \vec{B})\left(\vec{\nabla} \alpha_{1}\right)\right. \\
& \left.+\gamma_{1} k^{2} \vec{B}-i\left(\vec{\nabla} \beta_{1}\right) \times(\vec{k} \times \vec{B})-i\left(\vec{\nabla}\left(\beta_{1}+\gamma_{1}\right) \vec{k}\right\} \vec{B}\right] e^{i \vec{k} \cdot \vec{x}} \\
& R_{1}\left(\vec{A} e^{i k \cdot \vec{x}}\right)=-i \omega \theta_{0}\left(1-i \delta_{l k} t_{0} \omega\right) m_{1}(\vec{k} \vec{A}) e^{i \vec{k} \cdot \vec{x}}, \\
& S_{1}\left(C e^{i k \cdot \bar{x}}\right)=\left[-i \omega\left(1-i t_{0} \omega\right) \eta_{1} C e^{i \vec{k} \cdot \bar{x}}\right]-i C \vec{\nabla}\left\{v_{1} \vec{\nabla}\left(e^{i \vec{k} \cdot \bar{x}}\right)\right\} \\
& =-i\left[\omega\left(1-i t_{0} \omega\right) \eta_{1}+\left(\vec{\nabla} v_{1}+i v_{1} \vec{k}\right) \vec{k}\right] C e^{i \vec{k} \cdot \vec{x}} \\
& \text { and }\left\langle L_{1}\right\rangle=0 \text {. }
\end{aligned}
$$

Let us write

$$
\left(L_{0}^{-1} L_{1}\langle v\rangle\right)^{T}=e^{i \vec{k}_{2} \cdot \vec{x}} e^{i \vec{k}_{1} \cdot \vec{x}}\left(c_{1}+i d_{1}, c_{2}+i d_{2}\right),
$$

where $c_{i}, d_{i}$, are linear functions of $A_{1}, A_{2}, B_{1}, B_{2}$, and $B_{3}$. Then it can be shown as before that

$$
{\overline{\left(L_{0}^{-1} L_{1}\langle v\rangle\right)}}^{T}\left(L_{0}^{-1} L_{1}\langle v\rangle\right)=e^{-2(\operatorname{Im} k) x} \ell\left(A_{l}, B_{l}\right)
$$

where $\ell\left(A_{l}, B_{l}\right)$ is clearly a non-negative, bilinear function of $A_{l}$ and $B_{l}$. Hence the amount of dispersion of waves from the mean field comes out to be $\varepsilon e^{-(\operatorname{Im} k) x} \sqrt{\ell}$.

It may be concluded that the dispersion of the wave field from the mean field may be obtained following this procedure in many other cases examined by employing Keller's perturbation theory.

\section{The uncoupled problem}

This section proposes to outline, approximately following Chow [25] the method of examining the uncoupled random micropolar generalized-thermal problem. With this end in view the $\vec{u}$-term is dropped from the temperature Eq. (7), so that the reduced temperature equation becomes

$$
\eta(\vec{x})\left[\dot{\theta}(\vec{x}, t)+t_{0} \ddot{\theta}(\vec{x}, t)\right]=\vec{\nabla}\{\nu(\vec{x}) \vec{\nabla}[\theta(\vec{x}, t)]\}+q .
$$


The objective is to study the random elasticity-displacement Eq. (5) independently. The three dispersion equations (29)-(31) now become:

$$
\begin{gathered}
{\left[\rho_{0} \omega^{2} \vec{A}-\left(\lambda_{0}+\mu_{0}\right)(\vec{k} \vec{A}) \vec{k}-\left(\mu_{0}+\kappa_{0}\right) k^{2} \vec{A}\right]+i \kappa_{0}(\vec{k} \times \vec{B})-\varepsilon\left(i m_{2}+\omega m_{3}\right) C \vec{k}} \\
+\varepsilon^{2}\left[\omega \theta_{0}\left(1-\omega t_{0} \delta_{l k}\right) m_{2}\left(m_{2}-i \omega m_{3}\right)(\vec{k} \vec{A})\right] \int\left[\left(\vec{\nabla} G_{4}\right)\right] e^{-i \vec{k} \vec{r}} d \vec{r} \\
-\varepsilon^{2} \int\left[\begin{array}{l}
\left\langle\left[ M_{1}\left\{G_{0}\left(M_{1}^{\prime} \vec{A}+P_{1}^{\prime} \vec{B}+K_{1}^{\prime} C\right)+G_{1}\left(N_{1}^{\prime} \vec{A}+Q_{1}^{\prime} \vec{B}\right)\right\}\right.\right. \\
+P_{1}\left\{G_{2}\left(M_{1}^{\prime} \vec{A}+P_{1}^{\prime} \vec{B}+K_{1}^{\prime} C\right)+G_{3}\left(N_{1}^{\prime} \vec{A}+Q_{1}^{\prime} \vec{B}\right)\right\} \\
\left.\left.+K_{1} G_{4}\left(R_{1}^{\prime} \vec{A}+S_{1}^{\prime} C\right)\right]\right\rangle
\end{array}\right] e^{-i \vec{k} \cdot \vec{r}} d \vec{r}=0, \\
\quad i \kappa_{0}(\vec{k} \times \vec{A})-\left[\left(2 \kappa_{0}-\rho_{0} j \omega^{2}+\gamma_{0} k^{2}\right) \vec{B}+\left(\alpha_{0}+\beta_{0}\right)(\vec{k} \vec{B}) \vec{k}\right] \\
-\varepsilon^{2} \int\left[\begin{array}{l}
\left\langle\left[ N_{1}\left\{G_{0}\left(M_{1}^{\prime} \vec{A}+P_{1}^{\prime} \vec{B}+K_{1}^{\prime} C\right)+G_{1}\left(N_{1}^{\prime} \vec{A}+Q_{1}^{\prime} \vec{B}\right)\right\}\right.\right. \\
\left.\left.+Q_{1}\left\{G_{2}\left(M_{1}^{\prime} \vec{A}+P_{1}^{\prime} \vec{B}+K_{1}^{\prime} C\right)+G_{3}\left(N_{1}^{\prime} \vec{A}+Q_{1}^{\prime} \vec{B}\right)\right\}\right]\right\rangle
\end{array}\right] e^{-i \vec{k} \cdot \vec{r}} d \vec{r}=0
\end{gathered}
$$

and

$$
\left[i \omega\left(1-i t_{0} \omega\right) \eta_{0}-\nu_{0} k^{2}\right] C=0
$$

Simplified Case I. Equation (105) is easily solvable since $C \neq 0$. The first two equations can be analyzed as before or otherwise. Considering equations up to $\varepsilon$-order terms one gets

$$
\left[\rho_{0} \omega^{2} \vec{A}-\left(\lambda_{0}+\mu_{0}\right)(\vec{k} \vec{A}) \vec{k}-\left(\mu_{0}+\kappa_{0}\right) k^{2} \vec{A}\right]+i \kappa_{0}(\vec{k} \times \vec{B})-\varepsilon\left(i m_{2}+\omega m_{3}\right) C \vec{k}=0
$$

and

$$
i \kappa_{0}(\vec{k} \times \vec{A})-\left[\left(2 \kappa_{0}-\rho_{0} j \omega^{2}+\gamma_{0} k^{2}\right) \vec{B}+\left(\alpha_{0}+\beta_{0}\right)(\vec{k} \vec{B}) \vec{k}\right]=0 .
$$

The six equations are

$$
\begin{gathered}
{\left[\rho_{0} \omega^{2}-\left(\lambda_{0}+2 \mu_{0}+\kappa_{0}\right) k^{2}\right] A_{1}-\varepsilon\left(i m_{2}+\omega m_{3}\right) C k=0,} \\
{\left[\rho_{0} \omega^{2}-\left(\mu_{0}+\kappa_{0}\right) k^{2}\right] A_{2}+i \kappa_{0} k B_{3}=0,} \\
i \kappa_{0} k B_{2}=0
\end{gathered}
$$

and

$$
\begin{gathered}
{\left[\left(2 \kappa_{0}-\rho_{0} j \omega^{2}\right)+\left(\alpha_{0}+\beta_{0}+\gamma_{0}\right) k^{2}\right] B_{1}=0,} \\
\left(2 \kappa_{0}-\rho_{0} j \omega^{2}+\gamma_{0} k^{2}\right) B_{2}=0,
\end{gathered}
$$




$$
i \kappa_{0} k A_{2}-\left(2 \kappa_{0}-\rho_{0} j \omega^{2}+\gamma_{0} k^{2}\right) B_{3}=0 .
$$

It is clear that the amplitude ratio

$$
\frac{A_{1}}{C}=\frac{\varepsilon\left(i m_{2}+\omega m_{3}\right) k}{\rho_{0} \omega^{2}-\left(\lambda_{0}+2 \mu_{0}+\kappa_{0}\right) k^{2}}=O(\varepsilon)
$$

and is dependent upon expectation values of generalized thermal parameters $m$ and $m^{\bullet}=t_{1} m$. Moreover $A_{1}$ is finite but small such that $A_{1}=O(\varepsilon)$. Also $B_{2}=0$ indicates that there is no propagation along $B_{2}$. Further $B_{1} \neq 0$ and $B_{1} \equiv$ finite, indicates that waves with wave number given by

$$
k^{2}=\frac{\rho_{0} j \omega^{2}-2 \kappa_{0}}{\alpha_{0}+\beta_{0}+\gamma_{0}}
$$

propagate in the medium. Finally the coupled dispersion equation for waves propagating along $A_{2}$ and $B_{3}$ directions is represented by

$$
\begin{aligned}
& \frac{A_{2}}{B_{3}}=-\frac{i \kappa_{0} k}{\rho_{0} \omega^{2}-\left(\mu_{0}+\kappa_{0}\right) k^{2}}=\frac{2 \kappa_{0}-\rho_{0} j \omega^{2}+\gamma_{0} k^{2}}{i \kappa_{0} k}, \\
& {\left[\rho_{0} \omega^{2}-\left(\mu_{0}+\kappa_{0}\right) k^{2}\right]\left[2 \kappa_{0}-\rho_{0} j \omega^{2}+\gamma_{0} k^{2}\right]=\kappa_{0}^{2} k^{2} .}
\end{aligned}
$$

Case II. Unlike Chow ([25], cf. Eq. (4.3)), we retain the $\varepsilon$-order term in (31) to get

$$
\left[i \omega\left(1-i t_{0} \omega\right) \eta_{0}-\nu_{0} k^{2}\right] C-\varepsilon \omega \theta_{0}\left(1-i \omega t_{0} \delta_{l k}\right) m_{2}(\vec{k} \vec{A})=0 .
$$

Then Eqs. (103), (104), and (105) can be analyzed as in Sec. 4 or otherwise.

\section{Summary and conclusions}

1. The integrand of $a_{11}$ only is dependent on generalized thermal parameters including thermomechanical auto-correlation function and the cross-correlation function between thermomechanical and generalized thermomechanical coupling parameters. This dependence holds good both for L-S and G-L thermoelastic fields.

2. Numerical solutions can be easily obtained by setting

$$
R_{m m}(r)=\left\langle\rho_{1}^{2}\right\rangle e^{-\frac{r}{b}}, R_{m m}(r)=\left\langle\rho_{1}^{2}\right\rangle e^{-\frac{r}{b}} .
$$


3. On the other hand integrands of $a_{22}, a_{62}, b_{23}$, and $b_{63}$ are clearly independent of auto- and cross-correlation functions $R_{m m}(r)$ and $R_{m m} \cdot(r)$.

4. The three vector wave equations considered up to first order perturbation only leads to the dispersion equation for the wave propagation in the deterministic micropolar elastic medium. However effects of generalized thermal field are discernible only if the second order perturbation term is retained in this dispersion equation. This will be evident from the Eq. (48) of Sec. 4.

5. Equation (64) shows that longitudinal type displacement waves get modified due to random fluctuation of inhomogeneities of the micropolar medium under generalized thermoelasticity such that the wave number is increased by $\varepsilon^{2} \frac{\delta_{l}}{2 k_{l}}$, where $\delta_{l}$ is a function of $R_{m m}(r)$ and $R_{m m} \bullet(r)$. These results are valid for both L-S and G-L generalized thermoelasticity.

6. Equation (68) shows that the change in wave number $\delta_{l}$ depends upon the random variation of the generalized thermal field and measured in terms of statistical means, $m_{2}$ and $m_{3}$, for both L-S and G-L theories. For L-S, the change depends upon $m_{2}$ only, and for G-L upon $m_{2}$ and $m_{3}$.

7. Equation (100) shows that the dispersion $(\sqrt{\text { variance }})$ of the field from the mean field is a small quantity of order $\varepsilon$ and that it decays as the wave propagates in those cases where $\operatorname{Im} k>0$. This result holds good only if $m_{3}=t_{0} m_{2}$.

8. It is quite clear from Eqs. (70) and (75) that longitudinal type microrotation waves propagate in the random generalized thermoelastic micropolar medium but are scarcely affected by the random inhomogeneities of the medium, at least up to the domain of second order perturbation. In this case the amplitude $B_{1}$ is important in comparison to the amplitudes $A_{1}, A_{2}, B_{2}$, and $B_{3}$.

The study of micropolar materials and micropolar generalizedthermoelasticity in particular has been enormously drawing the interest of applied mathematicians and engineers in recent times. Micropolar materials include fibrous and granular or composite substances. The domain of micropolarity is therefore expanding in time. 
The study of micropolar properties in relation to wave propagation in coupled media therefore has been gaining importance from the perspective of probable applications in industry, engineering and to a great extent in earthquake prediction and seismology. Research studies are being carried out through various methodologies. The present paper has introduced the smooth perturbation technique [21] in examining the phenomenon of wave propagation in an infinite random generalized thermoelastic micropolar medium. In future certain other methods, viz., iterative perturbation method [31] or the method of scatters [37] may be employed in measuring effects of random variation of parameters. The domain decomposition method developed by Adomian [43] may also be employed to study micropolar elasticity in coupled dynamic problems. There is possibility of solving micropolar coupled elastic problems with the help of fractional calculus theories $[44,45]$.

Acknowledgement The authors sincerely thank the reviewers for making a number of excellent suggestions towards improving the manuscript.

Received 11 October 2016

\section{References}

[1] ERIngen A.C.: Linear theory of micropolar elasticity. J. Math. Mech. 15(1966), 6, 909-922.

[2] ERINGen A.C.: Theory of thermo-microstretch elastic solids. Int. J. Eng. Sci. 28(1990), 12, 1291-1301.

[3] ERINGen A.C.: Theory of thermo-microstretch fluids and bubbly liquids. Int. J. Eng. Sci. 28(1990), 2, 133-143

[4] ERIngen A.: Microcontinuum Field Theories. Springer Science + Business Media, New York 1999.

[5] MaRin M.: Some basic theorems in elastostatics of micropolar materials with voids. J. Comput. Appl. Math. 70(1996), 1, 115-126.

[6] MaRin M.: A domain of influence theorem for microstretch elastic materials. NonLinear Analysis: Real World Applications 11(2010), 5, 3446-3452.

[7] Marin M., Lupu M.: On harmonic vibrations in thermoelasticity of micropolar bodies. J. Vib. Control 4(1998), 5, 507-518.

[8] Marin M., Marinescu C.: Thermoelasticity of initially stressed bodies, asymptotic equipartition of energies. Int. J. Eng. Sci. 36(1998), 1, 73-86. 
[9] KUMAR R.: Wave propagation in micropolar viscoelastic generalized thermoelastic solid. Int. J. Engg. Sci. 38(2000), 1377-1395.

[10] Singh B.: Wave propagation in an anisotropic generalized thermoelastic solid. Indian J. Pure Appl. Math. 34(2003), 10, 1479-1485.

[11] Singh B., Kumar R.: Reflection and Refraction of plane waves at an interface between micropolar elastic solid and viscoelastic solid. Int. J. Eng. Sci. 36(1998), 2, 119-135.

[12] Kumar R., Deswal S: Surface wave propagation in a micropolar thermoelastic medium without energy dissipation. J. Sound Vibration 256(2002), 1, 173-178.

[13] Kumar R., Singh B.: Wave propagation in a micropolar generalized thermoelastic body with stretch. Proc. Indian Acad. Sci. (Math. Sci), 106(1996), 2, 183-199.

[14] Kumar R., Tomar S.K.: Propagation of micropolar waves at boundary surface. Indian J. Pure Appl. Math. 27(1996), 8, 821-835.

[15] Aound M.: The coupled theory of micropolar thermoelastic diffusion. Acta Mechanica 208(2009), 181-203.

[16] Suiker A.S.J., Borst R. DE, Chang C.S.: Micro-mechanical modelling of granular material: Part I: Derivation of a second-gradient micro-polar constitutive theory. Part II: Plane wave propagation in infinite media Acta Mechanica 149(2001), 1612001.

[17] Majewski E.: Earthquake Source Asymmetry, Structural Media and Rotation Effects, Chapter 19 (R.Teisseyre, M. Takeo, and E. Majewski, Eds.). Springer Verlag, 2006.

[18] Mitra M, Bhattacharyya R.K.: On wave propagation in a random micropolar thermoelastic medium, second soments and associated Green's tensor. Wave Random Complex 25(2015), 4, 506-535.

[19] Lord H.W., Shulman Y.A.: Generalized dynamical theory of thermoelasticity. J. Mech. Phys. Solids 15(1967), 299-309.

[20] Green A.E.,Lindsay K.A.: Thermoelasticity. J. Elasticity 2(1972), 1-7.

[21] Ignaczak J., Ostoja-Starzewski M.: Thermoelasticity with Finite Wave Speeds. Chap. 6. Oxford University Press, Oxford 2010.

[22] Keller J.B.: Stochastic equations and wave propagation in random media. Proc. Symp. App. Math. 16(1964), 145-170.

[23] Karal F.C., Keller J.B.: Elastic, electro-magnetic and other waves in a random medium. J. Mathematical Phys. 5(1964), 537-547.

[24] Keller J.B., Karal F.C.: Effective dielectric constant, permeability and conductivity of a random medium and the velocity and attenuation of coherent waves. J. Math. Phys. 7(1966), 661-670.

[25] Chow P.L.: Thermoelastic wave propagation in a random medium and some related problems. Int. J. Eng. Sci 11(1973), 953-971.

[26] Chen Y.M., Tien C.L.: Penetration of temperature waves in a random medium. J. Maths. Physics XLVI, 2, 1967. 
[27] Bhattacharyya R.K.: On wave propagation in a Random magneto-thermoviscoelastic medium. Indian J. Pure appl. Math. 17(1986), 705-725.

[28] BhattachaRyya R.K.: On reflection of waves from the boundary of a random elastic semi-infinite medium. Pure App. Geophysics (PAGEOPH) 146(1996), 3-4, 677-688.

[29] BerA R.K.: Propagation of waves in a random magneto-thermoelastic medium. Computers and Mathematics with Applications 36(1998), 9, 85-102.

[30] Chernov L.A.: Wave Propagation in a Random Medium. McGraw Hill, 1960.

[31] Beran M.J., MCCoy J.J.: Mean field variation in random media. Quart. App. Math. 28(1970), July, 245-258.

[32] Beran M.J., Frankenthal S., Deshmukh V., Whitman A.M.: Propagation of radiation in time-dependent three-dimensional random media. Wave Random Complex 18(2008), 3, 435-460.

[33] SовсZYк K.: Elastic wave propagation in a discrete random medium. Acta Mechanica 25(1976), 13-18.

[34] Wenzel A.R.: Radiation and attenuation of waves in a random medium. J. Acoust Soc. Am. 71(1982), 1, 26-35.

[35] Sobczyk K., Wedrychowicz S., Spencer B.F. JR: Dynamics of structural systemswith spatial randomness. Int. J. Solids Struct. 33(1996), 11, 1651-1669.

[36] Frankenthal S., Beran M.J.: Propagation in one-dimensionally stratified timeindependent scattering media. Wave Random Complex 17(2007), 2, 189-212.

[37] Uscinski B.J.: Intensity fluctuations in a moving random medium. Wave Random Complex 15(2005), 4, 437-450.

[38] Frisch U.: Wave Propagation in Random Media. In: Probabilistic Methods in Applied Mathematics (A.T. Bharucha-Reid, Ed.), 1, 76-198, Academic Press, New York 1968

[39] Chen K.K., Soong T.T.: Covariance properties of waves propagating in a random medium. J. Acoustical. Society of America 49(1971), 5(2), 1639-1642.

[40] Soong T.T.: Random Differential Equations in Science and Engineering. Academic Press, New York 1973.

[41] Ishimaru A.: Wave Propagation and Scattering in Random Media, Oxford University Press, Oxford 1978.

[42] Choudhury M., Basu U., Bhattacharyya R.K.: Wave propagation in a rotating randomly varying granular generalized thermoelastic medium. Comput. Math. Appl. 70(2015), 2803-2821.

[43] Adomian George: Non-Linear Stochastic Operator Equations. Academic Press, Harcourt Brace Jovanovich, New York, London 1986.

[44] Podlubny I.: Fractional Differential Equations. Academic Press. San Diego, New York, London 1999.

[45] Povstenko Y.Z.: Fractional heat conduction equation and associated thermal stress. J. Thermal Stresses 28(2004), 83-102.

[46] Lavoine J.: Methods de Calcul II, Calcul Symbolique Distributions et pseudofonctions. Centre National de la Recherche Scientifique, Paris 1959. 


\section{Appendix I}

\section{Components of the associated Green's tensor}

The $36+9$ components of the Green's tensor

$$
G=\left|\begin{array}{lll}
G_{1} & G_{2} & 0 \\
G_{3} & G_{4} & 0 \\
0 & 0 & G_{5}
\end{array}\right|
$$

where $G_{i}, i=1,2,3,4$, are $3 \times 3$ matrices, are then obtained and given by (for $\omega^{2}>\kappa_{0}\left(\kappa_{0}+2 \mu_{0}\right)$ :

$$
\left(G_{j}\right)_{k l}=\left(G_{j}\right)_{k k} \delta_{k l}
$$

with $\left(G_{1}\right)_{11}=\left(G_{1}\right)_{22}$ for $j=1,4$;

$$
\left(G_{j}\right)_{k l}=0 \quad \text { if }(k, l) \neq(1,2),(2,1)
$$

with $\left(G_{1}\right)_{12}=-\left(G_{1}\right)_{21}, j=2,3$;

and $\left(G_{3}\right)_{k l}=-\left(G_{2}\right)_{k l}$.

Components of $G_{5}$ have been defined in $(27)$ and (28).

The independent components $\left(G_{1}\right)_{11},\left(G_{1}\right)_{33},\left(G_{2}\right)_{12},\left(G_{4}\right)_{11}$ and $\left(G_{4}\right)_{33}$ are given by:

$$
\begin{aligned}
\left(G_{1}\right)_{11} & =\frac{i \kappa_{0}^{2}}{2 \pi \gamma_{0}\left(\mu_{0}+\kappa_{0}\right)^{2} r^{3}}\left[\frac{r^{2}-\left(i+k_{m} r\right)}{\left(k_{m}^{2}-k_{c}^{2}\right)\left(k_{m}^{2}-k_{s}^{2}\right)} e^{i k_{m} r}\right. \\
& \left.+\frac{r^{2}-\left(i+k_{c} r\right)}{\left(k_{c}^{2}-k_{m}^{2}\right)\left(k_{c}^{2}-k_{s}^{2}\right)} e^{i k_{c} r}+\frac{r^{2}-\left(i+k_{s} r\right)}{\left(k_{s}^{2}-k_{m}^{2}\right)\left(k_{s}^{2}-k_{c}^{2}\right)} e^{i k_{s} r}\right] \\
& -\frac{1}{4 \pi r^{3}}\left[\frac{r^{2} k_{s^{\prime}}^{2}+i r k_{s^{\prime}}-1}{\mu_{0} k_{s^{\prime}}^{2}} e^{i k_{s^{\prime}} r}-\frac{i k_{c^{\prime}} r-1}{\left(\lambda_{0}+2 \mu_{0}\right) k_{c^{\prime}}^{2}} e^{i k_{c^{\prime}} r}\right] \\
\left(G_{1}\right)_{33} & =\frac{-i \kappa_{0}}{\pi \gamma_{0}\left(\mu_{0}+\kappa_{0}\right)^{2} 2 r^{3}}\left[\frac{i+k_{m} r}{\left(k_{m}^{2}-k_{c}^{2}\right)\left(k_{m}^{2}-k_{s}^{2}\right)} e^{i k_{m} r}+\frac{i+k_{c} r}{\left(k_{c}^{2}-k_{m}^{2}\right)\left(k_{c}^{2}-k_{s}^{2}\right)} e^{i k_{c} r}\right. \\
& \left.+\frac{i+k_{s} r}{\left(k_{s}^{2}-k_{m}^{2}\right)\left(k_{s}^{2}-k_{c}^{2}\right)} e^{i k_{s} r}\right]+\frac{1}{4 \pi r^{3}}\left[\frac{2\left(i k_{s^{\prime}} r-1\right)}{\mu_{0} k_{s^{\prime}}^{2}} e^{i k_{s^{\prime}} r}-\frac{k_{c^{\prime}}^{2}{ }^{2}+2 i k_{c^{\prime}} r-2}{\left(\lambda_{0}+2 \mu_{0}\right) k_{c^{\prime}}^{2}} e^{i k_{c^{\prime}} r}\right] \\
\left(G_{2}\right)_{12} & =\frac{-k_{0}}{2 \pi \gamma_{0}\left(k_{c}^{2}-k_{s}^{2}\right)\left(\mu_{0}+k_{0}\right) r^{2}}\left[\left(i+k_{c} r\right) e^{i K_{c} r}-\left(i+k_{s} r\right) e^{i K_{s} r}\right]
\end{aligned}
$$

The other 9 components have been computed in the text. 


$$
\begin{aligned}
& \left(G_{4}\right)_{11}=\frac{-1}{2 \pi \gamma_{0}\left(\mu_{0}+\kappa_{0}\right)\left(k_{c}^{2}-k_{s}^{2}\right) r}\left[\left(\left\{\left(\mu_{0}+\kappa_{0}\right) k_{c}^{2}-\rho_{0} \omega^{2}\right) e^{i k \gamma}-\left\{\left(\mu_{0}+\kappa_{0}\right) k_{s}^{2}-\rho_{0} \omega^{2}\right) e^{i k \gamma}\right]\right. \\
& +\frac{1}{2 \pi \gamma_{0}\left(\mu_{0}+\kappa_{0}\right)\left(\alpha_{0}+\beta_{0}+\gamma_{0}\right) r^{3}}\left[\frac{\left[\left(\alpha_{0}+\beta_{0}\right)\left\{\left(\mu_{0}+\kappa_{0}\right) k_{c}^{2}-\rho_{0} \omega^{2}\right\}+\kappa_{0}^{2}\right]\left(1-i k_{c} r\right)}{\left(k_{c}^{2}-k_{s}^{2}\right)\left(k_{c}^{2}-k_{n}^{2}\right)} e^{i k_{c} r}\right. \\
& +\frac{\left[\left(\alpha_{0}+\beta_{0}\right)\left\{\left(\mu_{0}+\kappa_{0}\right) k_{s}^{2}-\rho_{0} \omega^{2}\right)+\kappa_{0}^{2}\right]\left(1-i k_{s} r\right)}{\left(k_{s}^{2}-k_{c}^{2}\right)\left(k_{s}^{2}-k_{n}^{2}\right)} e^{i k, r} \\
& \left.+\frac{\left[\left(\alpha_{0}+\beta_{0}\right)\left\{\left(\mu_{0}+\kappa_{0}\right) k_{x}^{2}-\rho_{0} \omega^{2}\right\}+\kappa_{0}^{2}\right]\left(1-i k_{n} r\right)}{\left(k_{n}^{2}-k_{c}^{2}\right)\left(k_{n}^{2}-k_{s}^{2}\right)} e^{i k_{n} r}\right] \\
& \left(G_{4}\right)_{33}=\frac{-1}{2 \pi \gamma_{0}\left(\mu_{0}+x_{0}\right)\left(k_{c}^{2}-k_{s}^{2}\right) r}\left[\left(\left(\mu_{0}+\kappa_{0}\right) k_{c}^{2}-\rho_{0} \omega^{2}\right) e^{i k_{c} \gamma}-\left\{\left(\mu_{0}+x_{0}\right) k_{s}^{2}-\rho_{0} \omega^{2}\right) e^{i k_{\gamma} r}\right] \\
& +\frac{1}{2 \pi \gamma_{0}\left(\mu_{0}+x_{0}\right)\left(\alpha_{0}+\beta_{0}+\gamma_{0}\right) r^{3}}\left[\frac{\left[\left(\alpha_{0}+\beta_{0}\right)\left\{\left(\mu_{0}+x_{0}\right) k_{c}^{2}-\rho_{0} \omega^{2}\right\}+x_{0}^{2}\right]\left(k_{c}^{2} r^{2}+2 i k_{c} r-2\right)}{\left(k_{c}^{2}-k_{s}^{2}\right)\left(k_{c}^{2}-k_{n}^{2}\right)} e^{i k_{r} r}\right. \\
& +\frac{\left[\left(\alpha_{0}+\beta_{0}\right)\left(\left(\mu_{0}+\kappa_{0}\right) k_{s}^{2}-\rho_{0} \omega^{2}\right)+\kappa_{0}^{2}\right]\left(k_{s}^{2} r^{2}+2 i k_{s} r-2\right)}{\left(k_{s}^{2}-k_{c}^{2}\right)\left(k_{s}^{2}-k_{n}^{2}\right)} e^{i k_{c} r} \\
& \left.+\frac{\left[\left(\alpha_{0}+\beta_{0}\right)\left(\left(\mu_{0}+x_{0}\right) k_{n}^{2}-\rho_{0} \omega^{2}\right)+x_{0}^{2}\right]\left(k_{n}^{2} r^{2}+2 i k_{n} r-2\right)}{\left(k_{n}^{2}-k_{c}^{2}\right)\left(k_{n}^{2}-k_{s}^{2}\right)} e^{i k_{k} r}\right]
\end{aligned}
$$

\section{Appendix II}

Method of forming products and taking expectation values $\left\langle M_{1} G_{0} M_{1}^{\prime}\right\rangle\left(\vec{A} e^{i \vec{k} \cdot \vec{x}^{\prime}}\right)$, etc.

$$
\begin{aligned}
& \left\langle N_{1} G_{0} M_{1}^{\prime}\right\rangle\left(\vec{A} e^{i \vec{k} \vec{x}^{\prime}}\right) \\
& =\kappa_{1} \vec{\nabla} \times G_{0}\left[-\left(\lambda_{1}^{\prime}+\mu_{1}^{\prime}\right)(\vec{k} \vec{A}) \vec{k}-\left(\mu_{1}^{\prime}+\kappa_{1}^{\prime}\right) k^{2} \vec{A}+\right. \\
& \left.i \vec{\nabla}^{\prime} \lambda_{1}^{\prime}(\vec{k} \vec{A})+i \vec{\nabla}^{\prime} \mu_{1}^{\prime} \times(\vec{k} \times \vec{A})+i\left[\vec{\nabla}^{\prime}\left(2 \overrightarrow{\mu^{\prime}}+\kappa_{1}^{\prime}\right) \vec{k}\right] \vec{A}+\rho_{1}^{\prime} \omega^{2} \vec{A}\right] e^{i \vec{k} \vec{x}^{\prime}} \\
& =\left[-\left(R_{* \lambda}+R_{* \mu}\right) A_{1} k^{2}\left(0, \frac{\partial\left(G_{0}\right)_{11}}{\partial z},-\frac{\partial\left(G_{0}\right)_{11}}{\partial y}\right)-k^{2}\left(R_{* \mu \mu}+R_{* *}\right)\left(-A_{2} \frac{\partial\left(G_{0}\right)_{22}}{\partial z}, A_{1} \frac{\partial\left(G_{0}\right)_{11}}{\partial z},\right.\right. \\
& \left.A_{2} \frac{\partial\left(G_{0}\right)_{22}}{\partial x}-A_{1} \frac{\partial\left(G_{0}\right)_{11}}{\partial y}\right)+i k A_{1}\left(\frac{\partial R_{* 1}}{\partial z^{\prime}} \cdot \frac{\partial\left(G_{0}\right)_{33}}{\partial y}-\frac{\partial R_{* 1}}{\partial y^{\prime}} \cdot \frac{\partial\left(G_{0}\right)_{22}}{\partial z},\right. \\
& \left.\frac{\partial R_{x-1}}{\partial x^{\prime}} \cdot \frac{\partial\left(G_{0}\right)_{11}}{\partial z}-\frac{\partial R_{x-1}}{\partial z^{\prime}} \cdot \frac{\partial\left(G_{0}\right)_{22}}{\partial x}, \frac{\partial R_{x \lambda}}{\partial y^{\prime}} \cdot \frac{\partial\left(G_{0}\right)_{22}}{\partial x}-\frac{\partial R_{x \lambda}}{\partial x^{\prime}} \cdot \frac{\partial\left(G_{0}\right)_{11}}{\partial y}\right)+i k A_{2}\left(\frac{\partial R_{x+4}}{\partial x^{\prime}} \cdot \frac{\partial\left(G_{0}\right)_{22}}{\partial z},\right. \\
& \left.A_{2} \frac{\partial\left(G_{0}\right)_{22}}{\partial x}-A_{1} \frac{\partial\left(G_{0}\right)_{11}}{\partial y}\right) \frac{\partial\left(2 R_{\kappa \mu}+R_{\kappa \kappa}\right)}{\partial x^{\prime}} \text {. }
\end{aligned}
$$




$$
\begin{aligned}
& \left.\frac{\partial R_{\kappa \mu}}{\partial y^{\prime}} \cdot \frac{\partial\left(G_{0}\right)_{11}}{\partial z},-\frac{\partial R_{\kappa \mu}}{\partial x^{\prime}} \cdot \frac{\partial\left(G_{0}\right)_{22}}{\partial x}-\frac{\partial R_{\kappa \mu}}{\partial y^{\prime}} \cdot \frac{\partial\left(G_{0}\right)_{11}}{\partial y}\right)+i k\left(-A_{2} \frac{\partial\left(G_{0}\right)_{22}}{\partial z}, A_{1} \frac{\partial\left(G_{0}\right)_{11}}{\partial z},\right. \\
& \left.+\omega^{2} R_{\kappa \rho}\left(-A_{2} \frac{\partial\left(G_{0}\right)_{22}}{\partial z}, A_{1} \frac{\partial\left(G_{0}\right)_{11}}{\partial z}, A_{2} \frac{\partial\left(G_{0}\right)_{22}}{\partial x}-A_{1} \frac{\partial\left(G_{0}\right)_{11}}{\partial y}\right)\right] e^{i \vec{k} \cdot x^{\prime}} .
\end{aligned}
$$

\section{Appendix III}

\section{A note on transforming to radial forms}

$\vec{r}=\vec{x}-\vec{x}^{\prime}=(\xi, \eta, \varsigma)=(r \cos \theta, r \sin \theta \cos \phi, r \sin \theta \sin \phi) ;$

$\frac{\partial}{\partial x}=\frac{\partial}{\partial \xi}, \frac{\partial}{\partial y}=\frac{\partial}{\partial \eta}, \frac{\partial}{\partial z}=\frac{\partial}{\partial \varsigma} ; \frac{\partial}{\partial x^{\prime}}=-\frac{\partial}{\partial \xi}, \frac{\partial}{\partial y^{\prime}}=-\frac{\partial}{\partial \eta}, \frac{\partial}{\partial z^{\prime}}=-\frac{\partial}{\partial \varsigma} ;$

$r^{2}=\xi^{2}+\eta^{2}+\varsigma^{2} ; \quad r \frac{\partial r}{\partial \xi}=\xi ; \therefore \frac{\partial r}{\partial \xi}=\frac{\xi}{r}, \frac{\partial r}{\partial \eta}=\frac{\eta}{r}, \frac{\partial r}{\partial \varsigma}=\frac{\varsigma}{r} ;$

$\frac{\partial}{\partial x}=\frac{\partial}{\partial \xi}=\left(\frac{\partial}{\partial r}\right) \frac{\partial r}{\partial \xi}=\frac{\xi}{r} \frac{\partial}{\partial r}=\cos \theta \frac{\partial}{\partial r} ;$

$\frac{\partial}{\partial y}=\frac{\eta}{r} \frac{\partial}{\partial r}=\sin \theta \cos \phi \frac{\partial}{\partial r} ; \frac{\partial}{\partial z}=\frac{\varsigma}{r} \frac{\partial}{\partial r}=\sin \theta \sin \phi \frac{\partial}{\partial r} ;$

$\frac{\partial^{2}}{\partial x^{2}}=\frac{\xi}{r} \frac{\partial}{\partial r}\left(\frac{\xi}{r} \frac{\partial}{\partial r}\right)=\cos ^{2} \theta \frac{\partial^{2}}{\partial r^{2}} ; \frac{\partial^{2}}{\partial y^{2}}=\frac{\eta}{r} \frac{\partial}{\partial r}\left(\frac{\eta}{r} \frac{\partial}{\partial r}\right)=\sin ^{2} \theta \cos ^{2} \phi \frac{\partial^{2}}{\partial r^{2}} ;$

$\frac{\partial^{2}}{\partial z^{2}}=\frac{\varsigma}{r} \frac{\partial}{\partial r}\left(\frac{\varsigma}{r} \frac{\partial}{\partial r}\right)=\sin ^{2} \theta \sin ^{2} \phi \frac{\partial^{2}}{\partial r^{2}}$.

1. $\int_{0}^{2 \pi} \cos ^{2} \phi d \phi=. \int_{0}^{2 \pi} \sin ^{2} \phi d \phi=\pi$;

2. $f(r)=\frac{\sin k r}{k r}$

3. $f^{\prime}(r)=\frac{d}{d(k r)} \frac{\sin k r}{k r} ; f^{\prime \prime}(r)=\frac{d^{2}}{d(k r)^{2}} \frac{\sin k r}{k r}$

4. $\int_{0}^{\pi} e^{-i k r \cos \theta} \sin \theta d \theta=2 \frac{\sin k r}{k r}=2 f(r)=\int_{-1}^{1} e^{-i k r x} d x$

5. $\int_{0}^{\pi} e^{-i k r \cos \theta} \sin \theta \cos \theta d \theta=2 i f^{\prime}(r)=\int_{-1}^{1} x e^{-i k r x} d x$

6. $\int_{0}^{\pi} e^{-i k r \cos \theta} \sin \theta \cos ^{3} \theta d \theta=-2 i f^{\prime \prime \prime}(r)$ 
7. $\int_{0}^{\pi} e^{-i k r \cos \theta} \sin ^{3} \theta \cos \theta d \theta=2 i\left[f^{\prime}(r)+f^{\prime \prime \prime}(r)\right]$

8. $\int_{0}^{\pi} e^{-i k r \cos \theta} \sin \theta \cos ^{2} \theta d \theta=-2 f^{\prime \prime}(r)$

9. $\int_{0}^{\pi} e^{-i k r \cos \theta} \sin ^{3} \theta d \theta=-2\left[f(r)+f^{\prime \prime}(r)\right]$

10. $\int_{0}^{2 \pi} \cos ^{3} \phi d \phi=\left|\sin \phi-\frac{1}{3} \sin ^{3} \phi\right|_{0}^{2 \pi}=0$.

\section{Appendix IV}

Finite parts of some integrals [46]

(i) Pf $\int_{0}^{\infty} \frac{e^{-p t}}{t} d t=-\log p-\gamma=-\log C p, \operatorname{Re} p>0$, where $\gamma=\log C=.577$ (Euler's constant) .

(ii) $\operatorname{Pf} \int_{0}^{\infty} \frac{e^{-p t}}{t^{2}} d t=p(\log p+\gamma-1)$.

(iii) $\int_{0}^{\infty} e^{-p t} \frac{\sin b t}{t} d t=\tan ^{-1} \frac{b}{p},|\arg p|<\pi / 2$.

(iv) $\operatorname{Pf} \int_{0}^{\infty} e^{-p t} \frac{\cos b t}{t} d t=-\frac{1}{2} \log \left(p^{2}+b^{2}\right)-\gamma,|\arg p|<\pi / 2$.

(v) $\operatorname{Pf} \int_{0}^{\infty} e^{-p t} \frac{\sin b t}{t^{2}} d t=\frac{-r}{1}[\theta \cos \theta+\{\log r-\Psi(2)\} \sin \theta,|\arg p|<\pi / 2$, $\theta=\tan ^{-1} \frac{b}{p}, r=\sqrt{p^{2}+b^{2}}$.

(vi) $\operatorname{Pf} \int_{0}^{\infty} e^{-p t} \frac{\cos b t}{t^{2}} d t=\frac{-r}{1}\{[\log r-\Psi(2)] \cos \theta-\theta \sin \theta\},|\arg p|<\pi / 2$, $\Psi(2)=-\gamma+1+\frac{1}{2}$ 